\title{
Characterization of a candidate dual AGN
}

\author{
D. Lena, ${ }^{1,2 \star}$ G. Panizo-Espinar, ${ }^{3}$ P. G. Jonker, ${ }^{1,2}$ M. A. P. Torres ${ }^{1,4,5}$ and M. Heida ${ }^{6}$ \\ ${ }^{1}$ SRON, Netherlands Institute for Space Research, Sorbonnelaan 2, NL-3584 CA Utrecht, the Netherlands \\ ${ }^{2}$ Department of Astrophysics/IMAPP, Radboud University Nijmegen, PO Box 9010, NL-6500 GL Nijmegen, the Netherlands \\ ${ }^{3}$ Facultad de Física, Universidad de La Laguna, Astrofísico Francisco Sánchez s/n, E-38206 La Laguna, Spain \\ ${ }^{4}$ Instituto de Astrofisica de Canarias, Calle Via Lactea s/n, E-38205 La Laguna, Tenerife, Spain \\ ${ }^{5}$ Dpto. de Astrofísica, Universidad de La Laguna, Astrofísico Francisco Sánchez s/n, E-38206 La Laguna, Spain \\ ${ }^{6}$ Cahill Center for Astronomy and Astrophysics, California Institute of Technology, 1200 California Boulevard, Pasadena, CA 91125, USA
}

Accepted 2018 April 30. Received 2018 April 30; in original form 2018 February 13

\begin{abstract}
We present Chandra and optical observations of a candidate dual active galactic nucleus (AGN) discovered serendipitously while searching for recoiling black holes via a cross-correlation between the serendipitous XMM source catalogue (2XMMi) and SDSS-DR7 galaxies with a separation no larger than 10 times the sum of their Petrosian radii. The system has a stellar mass ratio $M_{1} / M_{2} \approx 0.7$. One of the galaxies (Source 1 ) shows clear evidence for AGN activity in the form of hard X-ray emission and optical emission-line diagnostics typical of AGN ionization. The nucleus of the other galaxy (Source 2) has a soft X-ray spectrum, bluer colours, and optical emission-line ratios dominated by stellar photoionization with a 'composite' signature, which might indicate the presence of a weak AGN. When plotted on a diagram with X-ray luminosity versus [OIII] luminosity, both nuclei fall within the locus defined by local Seyfert galaxies. From the optical spectrum, we estimate the electron densities finding $n_{1}<27 \mathrm{e}^{-} \mathrm{cm}^{-3}$ and $n_{2}$ $\approx 200 \mathrm{e}^{-} \mathrm{cm}^{-3}$. From a 2D decomposition of the surface brightness distribution, we infer that both galaxies host rotationally supported bulges (Sérsic index $<1$ ). While the active nature of Source 1 can be established with confidence, whether the nucleus of Source 2 is active remains a matter of debate. Evidence that a faint AGN might reside in its nucleus is, however, tantalizing.
\end{abstract}

Key words: galaxies: active-galaxies: interactions-galaxies: nuclei.

\section{INTRODUCTION}

In the recipe of galaxy evolution, there are two ingredients of key importance: mergers and nuclear activity. Major mergers (defined as having a mass ratio $M_{2} / M_{1} \geq 0.3$ ) affect in dramatic ways the morphologies, star formation histories, dust content, gas distribution, and the growth of the supermassive black holes (SMBHs) residing in the nuclei of the merging galaxies.

Gravitational torques generated during the merger can drive gas from the outskirts of galaxies towards the inner regions (e.g. Hopkins et al. 2006). Gas might stall at the inner Lindblad resonance, typically $1 \mathrm{kpc}$ from the nucleus, producing starburst rings (e.g. Buta \& Combes 1996; Pérez-Ramírez et al. 2000). However, a chain of gravitational instabilities might be able to further transfer the gas towards the galactic nuclei, creating a reservoir that can fuel the SMBH (e.g. Hopkins \& Quataert 2010; Emsellem et al. 2015). The dark object will then reveal itself as an active galactic nucleus (AGN). Depending on the SMBH spin, mass, and the amount of gas available, the AGN might transfer mass and kinetic energy from the nucleus to the large-scale environment via outflows, regulating the star formation (e.g. Crenshaw, Kraemer \& George 2003). This interplay between host galaxy and AGN might originate the observed scaling relations between the SMBH and the bulge (e.g. Merritt 2013, section 2.4.5 of Heckman \& Best 2014).

While the merger proceeds, activity switches on and off (e.g. Van Wassenhove et al. 2012; Hickox et al. 2014; Schawinski et al. 2015). If both SMBHs are active, then the system, consisting of two interacting galaxies and two active SMBHs not yet gravitationally bound, becomes a 'dual AGN'.

In the meantime, the two SMBHs are believed to sink, because of dynamical friction, towards the minimum of the newly formed potential well. There the SMBHs will form a gravitationally bound system and, if dissipative mechanisms are efficient, their separation will decrease till the hardening of the orbit will be driven by the emission of gravitational waves (e.g. Merritt 2013, Ch. 8). The two black holes (BHs) will then merge, and asymmetries in the mass and spins of the SMBH binary will result in the asymmetric emission of gravitational waves (e.g. Bekenstein 1973). This process will remove linear momentum from the new SMBH delivering 
it a kick, the amplitude depending on the properties and orbital configuration of the SMBH binary (e.g. Campanelli et al. 2007; Tichy \& Marronetti 2007; Lousto \& Zlochower 2013). The rarity of recoiling SMBHs (very few seemingly convincing cases have been found to date, e.g. Civano et al. 2010, 2012), the small amplitude of AGNs spatially offset from their host nuclei (Lena et al. 2014), and the consistency between the SMBH occupation fraction at high and low redshift (Merritt \& Ferrarese 2001) suggest that recoils larger than a few $100 \mathrm{~km} \mathrm{~s}^{-1}$ are rare, perhaps because the spins of the merging SMBHs are nearly aligned, due to prolonged accretion.

While the broad picture sketched above might be correct, solid observational support for all the steps is still far from being achieved. For example, the role of galactic mergers in the onset of AGN activity has long been debated with studies giving very different results even in the local Universe (see Alexander \& Hickox 2012 for a review). Dual AGNs offer a snapshot of only one of the many phases taking place along the path to coalescence. Nevertheless, a thorough characterization of a large sample of dual AGNs (with a range of physical separations, with a range of activity levels) will boost our understanding of a number of processes, such as the dynamics of stars, gas, and SMBH during the merger (e.g. Blecha, Loeb \& Narayan 2013; Escala et al. 2013; Dotti, Merloni \& Montuori 2015; Khan et al. 2016), the growth of SMBHs (e.g. Callegari et al. 2011), the merger rate of galaxies and, to a lesser measure, the merger rate of SMBHs (important quantities to test cosmological models and to make prediction for observable gravitational wave events), and possibly we will gain insights into the expected electromagnetic counterparts of SMBH mergers (e.g. Milosavljević \& Phinney 2005; Haiman 2017).

The majority of studies aiming to identify dual AGNs begin by selecting galaxies displaying double peaked emission lines, a spectral feature that may indicate the presence of two interacting narrowline regions (NLRs), and therefore two AGNs (e.g. Zhou et al. 2004; Wang et al. 2009; Comerford et al. 2012; Barrows et al. 2013; Shi et al. 2014). However, it has long been known that double peaked emission lines could also originate from a broad range of phenomena, including unresolved rotation, non-gravitational motions in the ionized gas (i.e. inflows or outflows), structures in the NLR, interaction between jets and clouds, or one AGN illuminating two interacting galaxies (e.g. Heckman, Miley \& Green 1984; Komossa, Zhou \& Lu 2008; Xu \& Komossa 2009; Crenshaw et al. 2010; Gabányi et al. 2017). In recent years, integral field spectroscopy has shown at unprecedented level the complexity that the emission-line profiles can display within active galaxies (e.g. Schnorr Müller et al. 2011; Lena et al. 2015; Davies et al. 2017; Nevin et al. 2018). It is therefore clear that to confirm whether a candidate is a genuine dual AGN, further insights are needed into the origin of the emission-line profiles (e.g. McGurk et al. 2015; Müller-Sánchez et al. 2016), and into the nature of the galactic nuclei. Usually, this requires multiwavelength follow-up observations (e.g. Komossa et al. 2003; Fu et al. 2011; Koss et al. 2011a; Mazzarella et al. 2012; Liu et al. 2013; Gabányi et al. 2016; Hainline et al. 2016).

Attempting to bypass the ambiguous origin of the optical emission-line profiles, different approaches have been explored in the selection of dual AGNs: e.g. Koss et al. (2012) started from the sample of ultra-hard X-ray selected AGNs in Koss et al. (2011b) and looked for apparent companions within given radial and velocity bins. Recently, Satyapal et al. (2017) targeted optically obscured dual AGNs by selecting interacting galaxies displaying mid-infrared colours typical of AGNs.
In this paper, we present X-ray and optical follow-up for a candidate which we found serendipitously with still another method while searching for galaxies associated with single X-ray sources spatially offset from the optical hosts (possibly recoiling SMBHs). More specifically, in the Sloan Digital Sky Survey Data Release 7 (SDSS-DR7, Abazajian et al. 2009), we searched for interacting galaxies characterized by a maximum separation of 10 times the sum of their Petrosian radii (Petrosian 1976). Subsequently, we cross-correlated the sample with the XMM serendipitous source catalogue (2XMMi, Watson et al. 2009) looking for X-ray sources within 1.5 arcmin from the SDSS positions. Our approach yielded two candidates. The first one is the hyperluminous off-nuclear $\mathrm{X}$ ray source presented in Jonker et al. (2010, a candidate recoiling $\mathrm{SMBH})$. The second one is the candidate dual AGN presented in this work. The system consists of two galaxies (hereafter 'Source 1 ', the eastern and 'Source 2', the north-western, Fig. 1) with a projected separation $\Delta r=8.1$ arcsec; their SDSS IDs and coordinates are indicated in Table 1.

This object was also listed among the AGN pairs selected by Liu et al. 2011 on the basis of the SDSS-DR7 emission-line ratios.

Throughout the paper, we assume the cosmological parameters $H_{0}=70 \mathrm{~km} \mathrm{~s}^{-1} \mathrm{Mpc}^{-1}, \Omega_{m}=0.3$, and $\Omega_{v}=0.7$.

\section{OBSERVATIONS AND DATA REDUCTION}

\subsection{X-ray}

$\mathrm{X}$-ray imaging of the target was performed with the backilluminated chip (S3) of the Advanced CCD Imaging Spectrometer (ACIS, Garmire et al. 2003) of the Chandra X-ray Observatory (Weisskopf et al. 2000). A $9.93 \mathrm{Ks}$ exposure was taken in 'timed exposure' mode with telemetry formats set to 'very faint' (observations ID: 12143).

We reduced the data using $\mathrm{CIAO}^{1}$ (v4.8, Fruscione et al. 2006) and the calibration files CALDB v4.7.2. The data reduction was performed with the application of the CIAO tasks CHANDRA_REPRO, WAVDETECT, and WCS_UPDATE.

The script CHANDRA_REPRO takes in input the raw data and generates a bad pixel file, an event file, and optimizes spatial resolution via subpixel event repositioning (e.g. Li et al. 2004 and references therein). Afterwards, we used WAVDETECT (Freeman et al. 2002) for source detection and WCS_UPDATE to align the Chandra and SDSS world coordinate system (WCS).

For WAVEDETECT, we adopted a significance threshold of $1 \times 10^{-7}$. In the energy range $0.3-0.7 \mathrm{keV}, 10$ sources were found in the image that was searched, which corresponds to the section of the S3 CCD delimited by the image coordinates (3650:4550, 3800:4275). Because of the faintness of most sources, we chose only one for the WCS update, hereafter $\mathrm{x} 1$, which implies that we ignored any uncertainty on the satellite roll angle. The source $\mathrm{x} 1$ was detected by WAVEDETECT with a significance of 18 (the second most significant source detected, after one of the target AGNs) and it corresponds to the star SDSS J085319.63+162644.4, which also appears in Gaia data release 1 (DR1, Perryman et al. 2001; Gaia Collaboration 2016). Using the source position as given in Gaia-DR1, we obtained a bore-sight WCS correction of $\Delta$ (RA, Dec. $)=\left(-0^{\prime} .29,0^{\prime \prime} .36\right)$ \pm 0 '.08. The uncertainty on the bore-sight correction is dominated by the uncertainty in localizing the X-ray source on the ACIS-S3

${ }^{1}$ Chandra Interactive Analysis of Observations; http://cxc.harvard.edu/ciao/. 

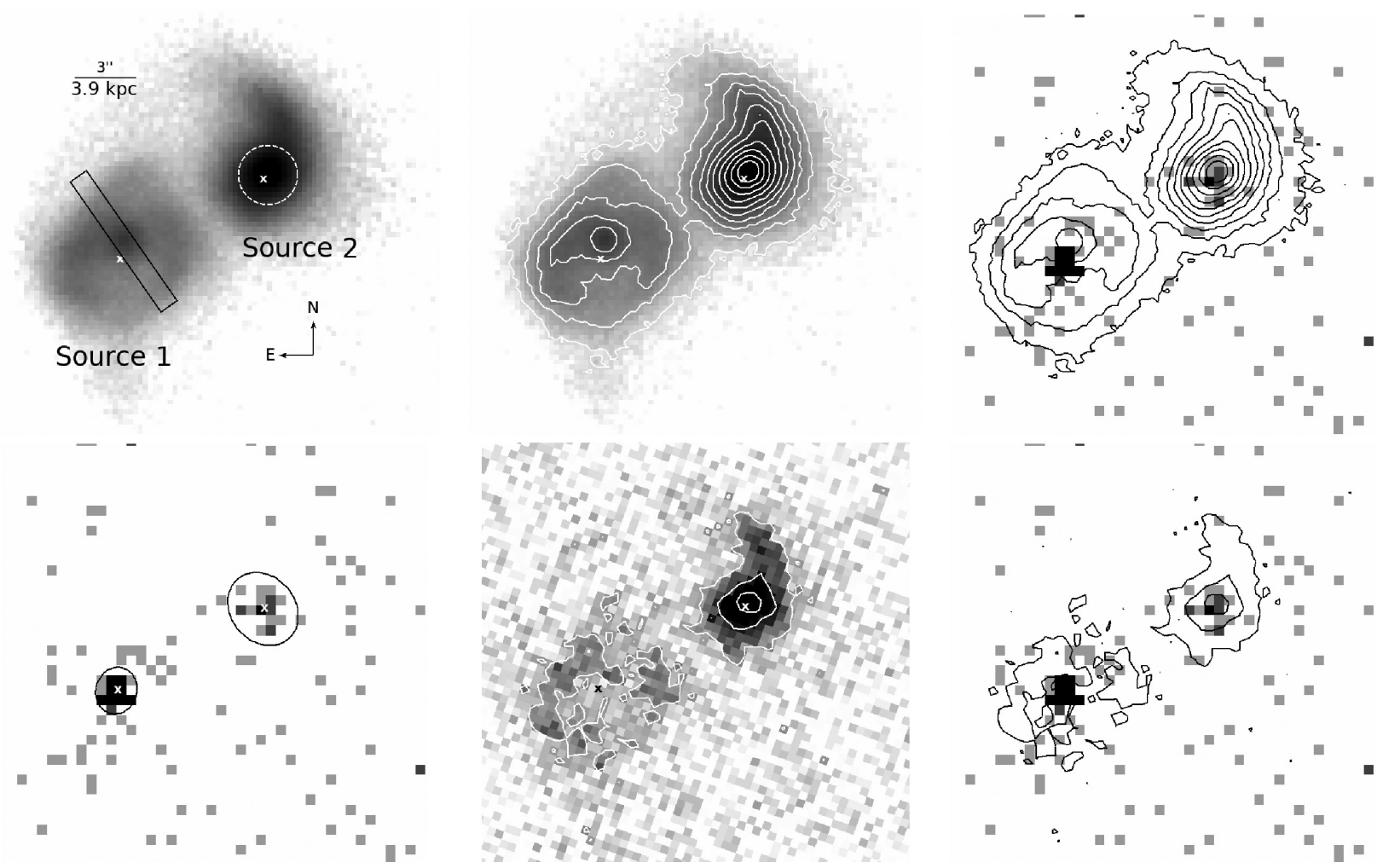

Figure 1. Top left: PanSTARRS-1 image of the two galaxies ( $r$ band; Chambers et al. 2016). The positions of the Chandra sources are indicated with a solid ' $\mathrm{x}$ ' of size 0.4 arcsec across. The dashed white circle represents position and size of the SDSS fibre used to obtain the spectrum. The solid black box represents orientation and width of the ACAM slit. Top centre: as in the left image, with a different colour scaling and asinh contours overlaid to highlight the morphology of the galaxies. The white crosses represent the Chandra sources. Top right: X-ray Chandra image with optical contours overlaid. Bottom left: X-ray Chandra image with extraction regions (black ellipses) and their centroids (white ' $\mathrm{x}$ '). Bottom centre: SDSS-DR13 image of the two galaxies ( $u$ band, Albareti et al. 2017) with asinh contours and Chandra sources indicated with ' $\mathrm{x}$ '. Bottom right: X-ray Chandra image with $u$-band SDSS optical contours.

Table 1. X-ray sources location. (1) Source ID; (2) SDSS-DR7 ID; (3-6) coordinates and centroid uncertainties for the Chandra sources after boresight correction. Uncertainties are in arcseconds and do not take into account the 0 '. 08 systematic uncertainty introduced by the bore-sight correction.

\begin{tabular}{lccccc}
\hline $\begin{array}{l}\text { Source } \\
(1)\end{array}$ & $\begin{array}{c}\text { SDSS name } \\
(2)\end{array}$ & $\begin{array}{c}\text { RA } \\
(3)\end{array}$ & $\begin{array}{c}\text { RA err } \\
(4)\end{array}$ & $\begin{array}{c}\text { Dec. } \\
(5)\end{array}$ & $\begin{array}{c}\text { Dec. err } \\
(6)\end{array}$ \\
\hline 1 & $\mathrm{~J} 085312.85+162616.0$ & 133.30362 & $00^{\prime \prime} 04$ & 16.43756 & $0^{\prime \prime} 06$ \\
2 & $\mathrm{~J} 085312.34+162619.4$ & 133.30153 & $0^{\prime \prime} 2$ & 16.43868 & $0^{\prime \prime} 1$ \\
\hline
\end{tabular}

CCD using the Chandra data; the uncertainty on the position of the reference star, as measured with Gaia, is of order 1 mas. We did not account for the star proper motion.

Proper motion and position are available for the star in UCAC4 (Zacharias et al. 2013); however, we deemed the derived correction to be less accurate: when propagating the UCAC4 position of the star with its proper motion to the epoch of Gaia-DR1, we obtained an offset of 0.3 and 0.'1 in RA and Dec. between this inferred position and the one in Gaia.

\subsection{Optical}

SDSS spectra are available for both galaxies. However, the fibre used to observe Source 1 was not centred on the nucleus of the galaxy, which is the region that we want to probe. For this reason, we obtained a new spectrum using ACAM (Benn, Dee \& Agócs 2008) on the William Herschel Telescope.
Observations were carried out on 2017 April 12. The slit was oriented at the parallactic angle, the width was 1 arcsec, and the seeing varied between 0.9 and 1.2 arcsec. We obtained two exposures of $900 \mathrm{~s}$ each, centred on the nucleus, using the grism V400. The nominal instrumental dispersion is $\Delta \lambda \approx 3.3 \AA \mathrm{pixel}^{-1}$. For a slit width of $1 \mathrm{arcsec}$, the predicted resolving power is $R=430$ at $5650 \AA$, or $\Delta \lambda=13.1 \AA$ and $\sigma=296 \mathrm{~km} \mathrm{~s}^{-1}$, which is consistent with the value that we measured from the [O I] $\lambda 5577$ sky-emission line (this value is likely an overestimate as we measured smaller velocity dispersions from the fit of the spectrum, Section 3.2). To perform flux calibration, we observed the standard star BD+08d2015 (spectral type G2V) immediately before the target.

Data reduction was performed using IRAF. ${ }^{2}$ The data were corrected for the bias, flatfielded, calibrated in wavelength, and background subtracted. Variance-weighted spectra were extracted using an aperture of diameter 3.5 arcsec centred on the nucleus, a heliocentric correction of $-28.29 \mathrm{~km} \mathrm{~s}^{-1}$ was applied, fluxes were calibrated, and the two exposures combined into an average spectrum.

From the RMS residual of the IRAF task REIDENTIFY, we estimated that the wavelength calibration is accurate to within $0.5 \AA$. Before

${ }^{2}$ IRAF (Image Reduction and Analysis Facility) is a general purpose software system for the reduction and analysis of astronomical data. It is distributed by the National Optical Astronomy Observatory, which is operated by the Association of Universities for Research in Astronomy (AURA) under a cooperative agreement with the National Science Foundation. 
Table 2. Best-fitting parameters for the X-ray spectra. (1) Source ID; (2) column density, units $\mathrm{cm}^{-2}$; (3) power-law index; (4) observed flux; (5, 6) unabsorbed flux, untis: $\mathrm{erg} \mathrm{s}^{-1} \mathrm{~cm}^{-2}$; (7) observed luminosity; $(8,9)$ unabsorbed luminosity, untis: $\mathrm{erg} \mathrm{s}^{-1}$. Uncertainties represent 68 per cent confidence levels. The main source of uncertainty is the column density. The distances assumed to derive the fluxes, $D_{L, 1} \approx 291 \mathrm{Mpc}$ and $D_{L, 2} \approx 288 \mathrm{Mpc}$, have been derived in Section 4.1 . Observed fluxes in the band $2-10 \mathrm{keV}$ are consistent with the unabsorbed fluxes. ${ }^{\mathrm{a}}$ The flux in the $2-10 \mathrm{keV}$ range for Source 2 was extrapolated from the fit; no emission was observed above $2 \mathrm{keV}$.

\begin{tabular}{|c|c|c|c|c|c|c|c|c|c|}
\hline $\begin{array}{l}\text { Source } \\
\text { (1) }\end{array}$ & $\begin{array}{l}n_{\mathrm{H}} \\
(2)\end{array}$ & $\begin{array}{c}\Gamma \\
(3)\end{array}$ & $\begin{array}{c}F_{0.3-7 \mathrm{keV}}^{\mathrm{obs}} \\
(4)\end{array}$ & $\begin{array}{c}F_{0.3-7 \mathrm{keV}} \\
\quad(5)\end{array}$ & $\begin{array}{c}F_{2-10 \mathrm{keV}} \\
\text { (6) }\end{array}$ & $\begin{array}{l}L_{0.3-7 \mathrm{keV}}^{\mathrm{obs}} \\
\quad(7)\end{array}$ & $\begin{array}{l}L_{0.3-7 \mathrm{keV}} \\
\quad(8)\end{array}$ & $\begin{array}{l}L_{2-10 \mathrm{keV}} \\
\text { (9) }\end{array}$ & \\
\hline 1 & $5 \times 10^{22}$ & 1.9 & $\begin{array}{c}1.6_{-0.12}^{+0.2} \\
10^{-13}\end{array} \times$ & $\begin{array}{c}5.7_{-0.12}^{+0.2} \times \\
10^{-13}\end{array}$ & $\begin{array}{c}2.2_{-0.3}^{+0.2} \times \\
10^{-13}\end{array}$ & $\begin{array}{c}1.6_{-0.1}^{+0.2} \times \\
10^{42}\end{array}$ & $\begin{array}{c}5.8_{-0.1}^{+0.2} \\
10^{42}\end{array}$ & $\begin{array}{c}2.2_{-0.3}^{+0.2} \times \\
10^{42}\end{array}$ & \\
\hline 2 & $2 \times 10^{20}$ & $3.4_{-0.7}^{+0.8}$ & $\begin{array}{c}2.3_{-1}^{+2} \times \\
10^{-14}\end{array}$ & $\begin{array}{c}2.8_{-1}^{+2} \times \\
10^{-14}\end{array}$ & $\begin{array}{c}1.7_{-1.2}^{+1.3} \times \\
10^{-15}\end{array}$ & $2.3_{-1}^{+2} \times 10^{41}$ & $2.8_{-1}^{+2} \times 10^{41}$ & $\begin{array}{c}1.7_{-1.2}^{+1.3} \\
10^{40}\end{array} \times$ & a \\
\hline
\end{tabular}

flux calibration, a shift of $14 \AA$ was applied to the wavelength axis to account for a systematic shift in the wavelength calibration zero-point; the bias is likely due to the fact that the arc was not obtained immediately before/after the science exposure (a shift of this amplitude is typical for $\mathrm{ACAM}^{3}$ ). The amplitude of the shift was estimated from the wavelength of the sky lines [O I] $\lambda 5577.3$, $\mathrm{Na} I \lambda 5890$, and $[\mathrm{O}$ I $] \lambda \lambda 6300.3,6363.8$. The correction is estimated to be accurate to within $0.1 \AA$.

\section{DATA ANALYSIS AND RESULTS}

\subsection{X-ray sources and galactic nuclei}

As specified in Section 2, we aligned the coordinates of the Chandra data with the International Coordinate Reference System (ICRS) using a Gaia-DR1 source that was detected in X-rays on the same $\mathrm{CCD}$ as the target under consideration. Applying the coordinate correction allowed a more accurate comparison between optical and X-ray data showing that two X-ray sources are approximately coincident with the optical nuclei of the two galaxies (Fig. 1). The spatial offset between the X-ray source and the optical centroid is larger for Source 1; while the position of the X-ray centroid is consistent with the centroids derived from bluer optical images $(u$ and $g$ bands), the spatial offset increases at longer wavelengths, up to about $0^{\prime \prime} .8$.

The corrected coordinates for the X-ray sources are given in Table 1. Using WAVDETECT we counted $91 \pm 10 \mathrm{X}$-ray photons for Source 1, with a significance of 36 , and $21 \pm 5$ photons for Source 2, with a significance of 8 , and with uncertainties estimated assuming Poisson statistics. Counts were extracted using WAVDETECT from the elliptical regions shown in Fig. 1; they were centred on the X-ray sources with axes and position angles equal to 1".66, 1".77, $-15^{\circ}$ East of North for Source 1, and 2."45, 1".55, $40^{\circ}$ East of North for Source 2. Using the CIAO script fluximage, we obtained consistent source counts in the broad energy band $0.5-7 \mathrm{keV}$.

We extracted the background from a circular region of radius 40 arcsec in an area of the S3 CCD devoid of sources. The background amounts to $<1$ photon, which was subtracted from the counts given above.

To determine whether the X-ray emission is consistent with an AGN origin, we modelled the spectra of the sources, which were extracted from the regions specified above. To model the spectra, we used XSPEC ${ }^{4}$ (v.12.9, Arnaud 1996), and we adopted Cash statistics (Cash 1979) to fit an absorbed power law. Towards this end, we

\footnotetext{
${ }^{3} \mathrm{http}: / /$ www.ing.iac.es/Astronomy/instruments/acam/flexuretests.html

${ }^{4}$ https://heasarc.gsfc.nasa.gov/xanadu/xspec/
}

used the XSPEC function pegpwr $\times$ phabs, that is

$$
f(E)=K E^{-\alpha} \exp \left[-n_{\mathrm{H}} \sigma(E)\right],
$$

where $E$ is the energy, $K$ the unabsorbed flux, $\sigma(\mathrm{E})$ is the photoelectric cross-section (not including Thomson scattering), $n_{\mathrm{H}}$ is the equivalent hydrogen column (in units of $10^{22}$ atoms $\mathrm{cm}^{-2}$ ), $\alpha=\Gamma-1$ is the power-law index, with $\Gamma$ the 'photon index'.

From the fit, we derived the power-law index and the unabsorbed flux. For Source 2, the hydrogen column density was fixed to $n_{\mathrm{H}}=2 \times 10^{20} \mathrm{~cm}^{-2}$ (i.e. the Galactic value, Kalberla et al. 2005). As a similar assumption would produce a negative power law for Source 1, in this second case we determined the value of $n_{\mathrm{H}}$, which would produce a power-law index $\Gamma=1.9$, which is typical for AGNs (e.g. Risaliti \& Elvis 2004). This resulted in $n_{\mathrm{H}}=4.7 \times 10^{22} \mathrm{~cm}^{-2}$.

Results of the fit are summarized in Table 2. Spectra for the two sources along with the fits are presented in Fig. 2. The spectra have been rebinned for display purposes only.

\subsection{Fit of the optical spectrum}

The optical spectra of the two galaxies show a number of emission and absorption lines. We used PPXF v6.6.1 (Cappellari \& Emsellem 2004; Cappellari 2017) to fit the spectral region 5000-7500 $\AA$, which includes the emission lines $\mathrm{H} \beta$, [O III] $] \lambda 4959,5007$, $[\mathrm{O}$ I $] \lambda 6300, \mathrm{H} \alpha,[\mathrm{N}$ II] $\lambda$ and [S II]6716, 6731, and several stellar absorption features. The fit of the spectra was performed in two steps: initially, we fitted the absorption features (i.e. the stellar kinematics); afterwards, we held fix the parameters of the stellar kinematics, as recovered from the previous step, and we fitted the full spectrum, including the emission lines.

More specifically, to fit the stellar kinematics, we masked out any emission line, and the $\mathrm{Na}$ I $\lambda \lambda 5890,5896$ absorption lines, which are affected by the interstellar medium of the host galaxy. To reproduce the absorption line profiles, we used a subset of the MILES stellar templates (Sánchez-Blázquez et al. 2006) and we modelled the line profiles of the SDSS spectrum as Gauss-Hermite (GH) polynomials; as the SDSS wavelength calibration was performed using 'in vacuum' values, while the MILES templates used 'in air' values, we used the vac_to_air() function provided with the PPXF package to correct the wavelength axis of the SDSS spectrum to 'in air' values. Due to the lower resolution of the ACAM data, in this second case we assumed a Gaussian profile.

To fit the emission lines, we used four spectral components, each one modelled as a Gaussian. In particular, we used a spectral component to fit the $\mathrm{H} \beta$ emission line, one for the [O III] doublet, one for the $[\mathrm{O}$ I] doublet, and a fourth one to fit $\mathrm{H} \alpha,[\mathrm{N} \mathrm{II}]$, and [S II]. 

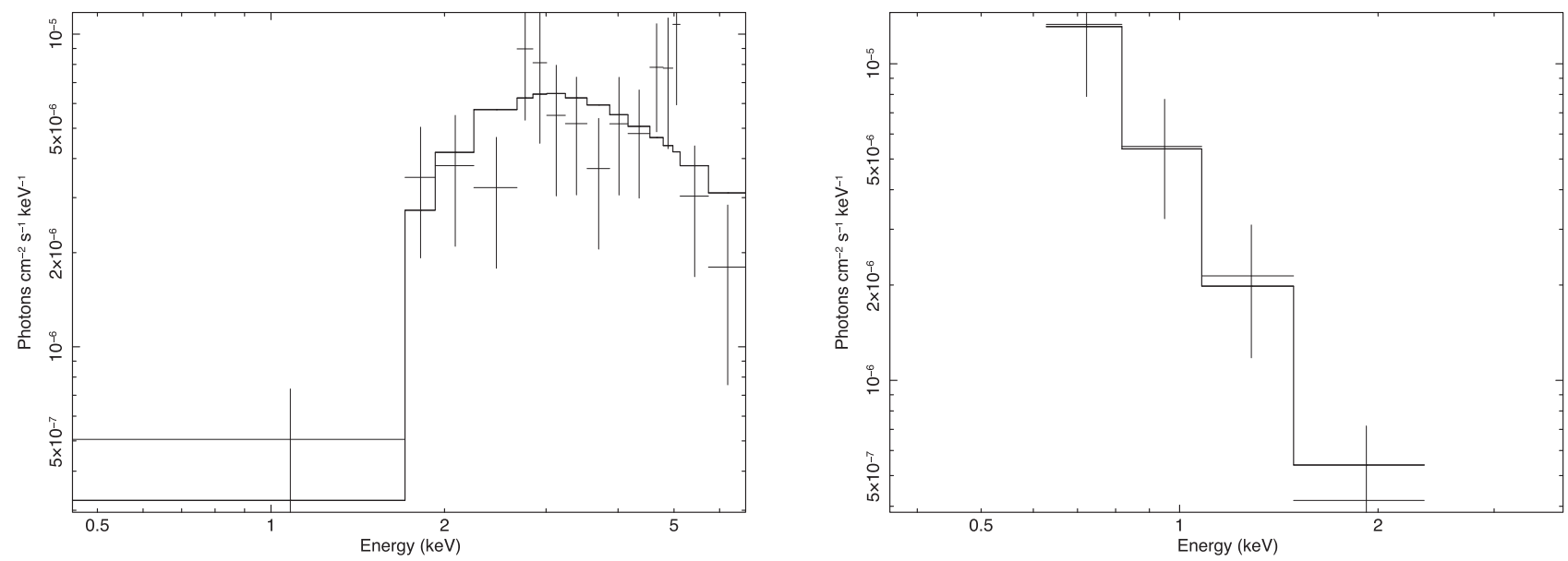

Figure 2. Observed Chandra spectra and XSPEC fit for Sources 1 and 2.

Table 3. Best-fitting parameters for the optical spectra. (1) Fitted spectral feature; (2) redshift; (3) velocity dispersion; (4) flux; (5) GH coefficients; (6) component ID. A ' $\mathrm{d}$ ' following the wavelength indicates that the flux refers to the emission-line doublet. Velocity dispersions for Source 1 were not corrected for the instrumental broadening because of the uncertain effective resolution of the instrument. Assuming an instrumental resolution $\Delta \lambda=13.1 \AA$ for a slit width of 1 arcsec, the instrumental velocity dispersions at the observed wavelengths are: $\sigma_{\star}=271 \mathrm{~km} \mathrm{~s}^{-1}$ (at $6150 \AA$ ) $, \sigma_{\mathrm{H} \beta}=322 \mathrm{~km} \mathrm{~s}^{-1}, \sigma_{[\mathrm{O} \mathrm{III]}}=313 \mathrm{~km} \mathrm{~s}{ }^{-1}$, $\sigma_{[\mathrm{O}]}=249 \mathrm{~km} \mathrm{~s}^{-1}, \sigma_{\mathrm{H} \alpha}=239 \mathrm{~km} \mathrm{~s}^{-1}, \sigma_{[\mathrm{N} \mathrm{II}]}=238 \mathrm{~km} \mathrm{~s}^{-1}, \sigma_{[\mathrm{S} \mathrm{I}]}=233 \mathrm{~km} \mathrm{~s}^{-1}$. Velocity dispersions for Source 2 are already corrected for instrumental broadening.

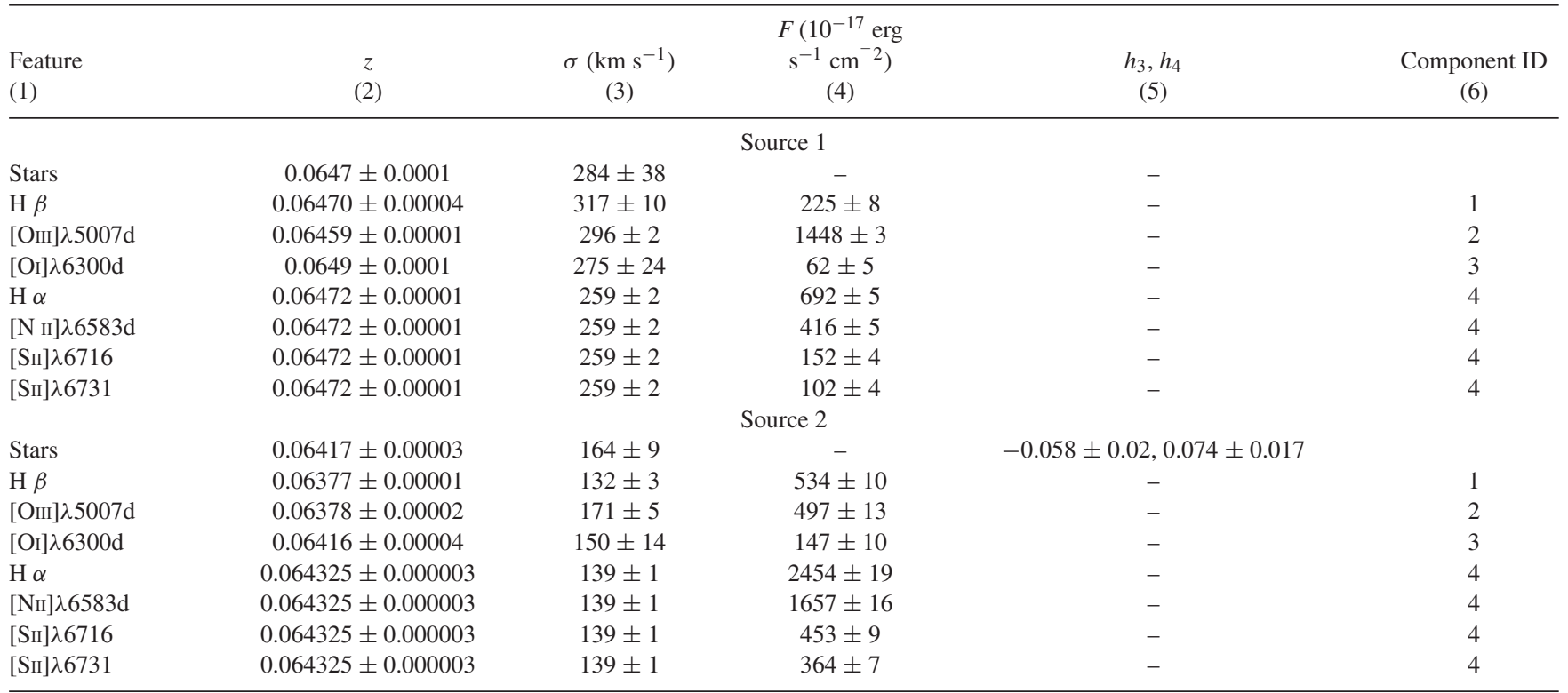

Default values were adopted for the PPXF parameters bias and regul_err, that is 0.386 and 0.0036 , respectively, for the SDSS spectrum; the same value was adopted for regul_err in the fit of the ACAM spectrum, while the bias value was irrelevant because a Gaussian profile was adopted. The noise array given to PPXF was the inverse square root of the inverse variance array. The fit of the SDSS spectrum took into account the intrinsic dispersion of every pixel using the information provided within the SDSS data set in the array $w$ disp. The resulting velocity dispersions were therefore corrected for instrumental broadening by PPXF itself. As a dispersion array for ACAM was not available, we performed the fit without any assumption on the instrumental dispersion, obtaining 'observed' velocity dispersions, i.e. not corrected for instrumental broadening. Results of the fit (redshift, velocity dispersion, fluxes, and GH coefficients) are shown in Table 3 and in Fig. 3.
We assessed the robustness of our solution against different values of the initial guesses (for redshift and velocity dispersion) and we checked how different values of the parameters bias and regul_err would affect the result. The solution showed negligible variations through the experiment. As expected, the most important variations were observed for the GH coefficients, which are biased towards larger or smaller values by the parameter bias. This variation in the $\mathrm{GH}$ coefficients produced a variation of $1 \mathrm{~km} \mathrm{~s}^{-1}$ in the stellar velocity dispersion.

To estimate the statistical uncertainties due to the fitting procedure, we performed a Monte Carlo simulation: to the observed spectrum, we added Gaussian noise with mean zero and standard deviation equal to the value of the noise array at that specific wavelength. We fitted the resulting spectrum and we repeated the process 100 times. From each fit, we derived redshift, velocity dispersion, 

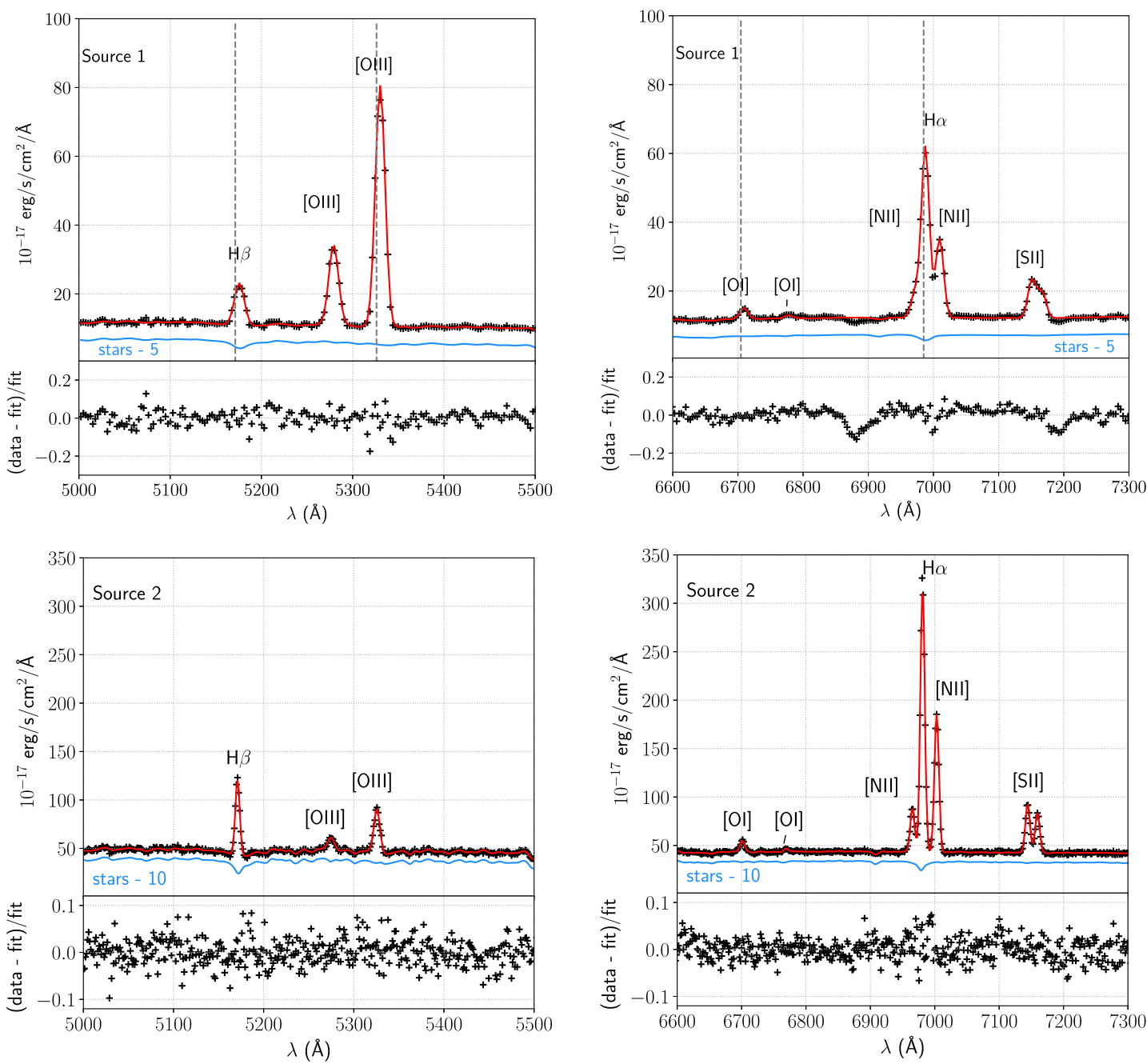

Figure 3. Top: ACAM spectrum for Source 1. Bottom: SDSS-DR12 spectrum for Source 2 (spectrum ID: 2431-53818-0452). Data points are plotted as black ' + ', the fit (including gas emission lines and the stellar continuum) is shown in red, the fitted stellar continuum is shown in blue and offset downward for clarity. To facilitate visual comparison, vertical dashed grey lines in the top panels indicate the peak of the corresponding emission lines derived for Source 2.

and flux (for the emission lines). The standard deviation of the parameter distribution was assumed to represent the wanted uncertainty, and it was derived using the NUMPY function $\operatorname{std}()$ with the parameters $d d o f()$ and dtype set to zero and np.float64, respectively. Results are indicated in Table 3.

\subsection{Electron density}

We estimated the electron density $\left(n_{\mathrm{e}}\right)$ using the [S II] $\lambda 6716 / 6731$ emission-line ratios (e.g. Osterbrock \& Ferland 2006, section 5.6).

From the flux values in Table 3, we derived the ratios $r_{1}=1.5 \pm 0.1$ and $r_{2}=1.24 \pm 0.05$. We assumed a temperature $T=10^{4} \mathrm{~K}$ and we used the IRAF task temden (De Robertis, Dufour \& Hunt 1987; Shaw \& Dufour 1995) to derive the corresponding densities.

The line ratio in Source 1 falls above the maximum theoretical limit, which corresponds to the low-density limit; we infer $n_{1, \mathrm{e}}<27$ $\mathrm{e}^{-} \mathrm{cm}^{-3}$. In Source 2, we find $n_{2, \mathrm{e}}=190 \pm 60 \mathrm{e}^{-} \mathrm{cm}^{-3}$.

Star-forming galaxies tend to have lower densities than Seyferts and low-ionization nuclear emission-line regions (LINERs; Ho 1996). For a sample of local $(0.04<z<0.1)$ star-forming galaxies, Sanders et al. (2016) obtained a median [S II] line ratio of 1.41 (corresponding to $n_{\mathrm{e}}=26 \mathrm{e}^{-} \mathrm{cm}^{-3}$ with their prescription) and they observed line ratios in the range $0.9<r<2.1$ (corresponding to densities $n_{\mathrm{e}} \lesssim 600 \mathrm{e}^{-} \mathrm{cm}^{-3}$ ). In the nuclei of local Seyferts, electron densities span the whole sensitivity range of the [S II] doublet $\left(n_{\mathrm{e}}<1500 \mathrm{e}^{-} \mathrm{cm}^{-3}\right)$, with typical values of a few hundreds electrons per $\mathrm{cm}^{-3}$ (e.g. Bennert et al. 2006; Lena et al. 2016; Brum et al. 2017). For the Palomar Survey of Nearby Galaxies sample, Ho (1996) quoted a median value for Seyfert galaxies of $n_{\mathrm{e}}=290$ $\mathrm{e}^{-} \mathrm{cm}^{-3}$.

\subsection{Optical and mid-infrared colours}

An optical colour map of the system was produced using the $g$ - and $y$-band PanSTARRS images (Chambers et al. 2016). The colour map was computed as

$g-y=-2.5 \log _{10} \frac{f_{g}}{f_{y}}+m_{0, g}-m_{0, y}$,

where $f=$ counts/exposure time is the linear pixel flux (as obtained for the cutout images from the PanSTARRS archive $\left.{ }^{5}\right) ; m_{0, g}$ and $m_{0, y}$

\footnotetext{
${ }^{5} \mathrm{http}: / /$ pslimages.stsci.edu/cgi-bin/ps1cutouts
} 

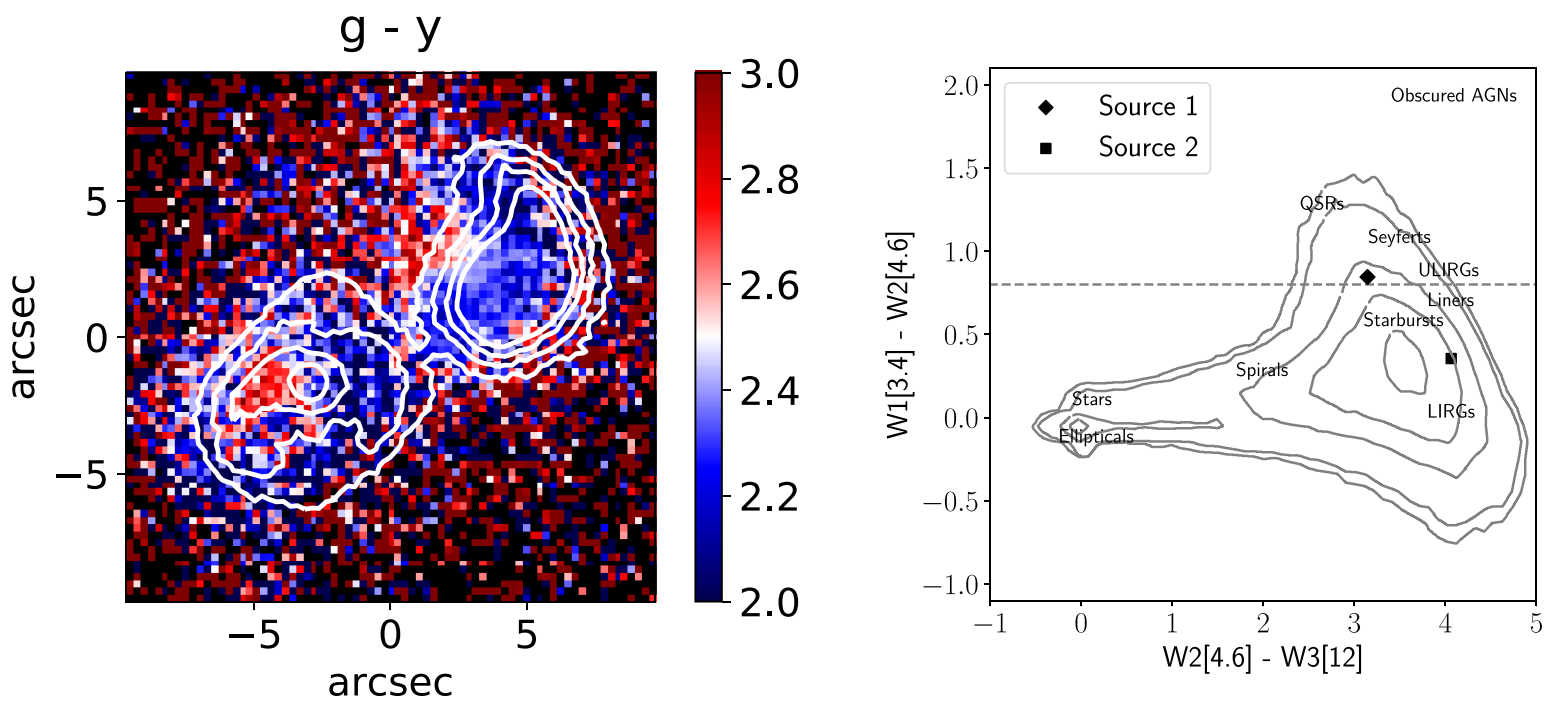

Figure 4. Left: optical colour map $(g-y)$ with $r$-band contours to highlight the most prominent photometric features. The innermost contours have been removed from Source 2 for clarity. Right: WISE colours overlaid on the colour-colour plot of Massaro et al. (2011). The contours represent isodensity curves for approximately $450000 \mathrm{WISE}$ sources. The location of different classes of objects is annotated. The horizontal line at $(\mathrm{w} 1-\mathrm{w} 2)=0.8$ indicates the threshold proposed by Stern et al. (2012) between AGN candidates and non-active galaxies. Uncertainties are smaller than the symbol size.

are the magnitude zero-points, which in the cutout images are both set to 25. The resulting map is shown in Fig. 4.

There is tentative evidence for resolved structures: the bar-like feature in Source 1 shows redder colours eastward of the nucleus. Source 2 also shows a gradient with bluer colours, on average, in the tail.

To derive the mid-infrared colours, we used photometric data from the 'All WISE Source Catalogue' ${ }^{6}$ Results are indicated in the right-hand panel for Fig. 4, where the colours for Source 1 and Source 2 are plotted on the colour-colour diagram of Massaro et al. (2011). Source 1 falls near the locus of Seyfert galaxies and above the threshold proposed by Stern et al. (2012) for the identification of candidate AGNs (i.e. $w 1-w 2 \geq 0.8$ ). Source 2 falls in between the locus of Starbursts and Luminous InfraRed Galaxies (LIRGs).

\subsection{D decomposition}

We used Galfit (v3.0.5, Peng et al. 2002, 2010) to perform a decomposition of the $2 \mathrm{D}$ surface brightness profile as traced by the old stellar population; towards this goal, we used the PanSTARRS $i$-band image.

To model Source 1, we adopted a compact Sérsic profile, which we interpret as a bulge, a truncated Sérsic profile for the dumbbelllike feature, and a distorted exponential disc for the faint stellar envelope and the irregularities associated with the 'dumbbell'.

The main body of Source 2 was modelled with a Sérsic profile and a nuclear point source (NPS); the 'tail' was modelled with a Sérsic component. An additional Sérsic profile was used to model a nearby faint galaxy located northward of the main targets, three other nearby objects were modelled as unresolved sources.

We derived a semi-empirical point spread function (PSF) by fitting to a nearby bright star (RA, Dec.: 08:53:09.30, +16:25:50.2)

${ }^{6}$ http://irsa.ipac.caltech.edu/cgi-bin/Gator/nph-scan?submit=Select\&projs hort=WISE the model adopted by the PanSTARRS team ${ }^{7}$

$$
\begin{aligned}
f(z) & =A \times\left(1+k z+z^{1.67}\right)^{-1}, \\
z & =\left(\frac{x^{2}}{2 \sigma_{x x}^{2}}+\frac{y^{2}}{2 \sigma_{y y}^{2}}+\sigma_{x y} x y\right),
\end{aligned}
$$

obtaining $k=0.6, \sigma_{x x}=1.83, \sigma_{y y}=1.82$, and $\sigma_{x y}=-0.01$. A model PSF was built (setting the amplitude $A=1$ ) and given in input to GALFIT. Both PSF image and convolution box were $400 \times 400$ pixels.

The resulting model and residuals are presented in Fig. 5. Subcomponents and best-fitting parameters are presented in Appendix A.

\subsection{Stellar mass ratio}

To estimate the total stellar mass ratio, we used the $r$ and $i$ mean Kron magnitudes (Kron 1980) from the PanSTARRS catalogue ${ }^{8}$ in conjunction with the scaling relation of Bell et al. (2003):

$\log _{10} \frac{M_{\star}}{M_{\odot}}=-0.4\left(M_{i}-M_{i, \odot}\right)+0.006+1.114(r-i)$,

where $M_{i}$ is the absolute $i$-band magnitude of the galaxy under consideration, $M_{i, \odot}=4.53$ (Blanton \& Roweis 2007) is the absolute AB magnitude of the Sun in the $i$-band, ${ }^{9}$ and $r$ and $i$ are the Kron magnitudes of our targets. Using the values in Table 4, we obtained $\log \left(M_{1, \star} / M_{\odot}\right)=10.7$ and $\log \left(M_{2, \star} / M_{\odot}\right)=10.9$, which correspond to a mass ratio $M_{1} / M_{2}=0.7$.

We preferred not to use magnitudes estimated via GALFIT because of a low-luminosity component (component \#4 in Fig. A2), which

\footnotetext{
${ }^{7}$ https://outerspace.stsci.edu/display/PANSTARRS/PS1+PSF+photometr $\mathrm{y}+$ of +detections\#PS1PSFphotometryofdetections-ThePSFModel ${ }^{8} \mathrm{http}: / /$ archive.stsci.edu/panstarrs/search.php

${ }^{9}$ The Sun magnitude provided by Blanton \& Roweis (2007) for the SDSs magnitude system was transformed to the PanSTARRS1 system using the linear relation provided by Tonry et al. (2012), which is $\left(i_{\mathrm{P} 1}-i_{\mathrm{SDSS}}\right)=0.004-0.014(g-r)$ SDSS , with $M_{i, \odot}=4.53, M_{g}, \odot=5.12$, and $M_{r, \odot}=4.64$. The resulting correction is negligible.
} 

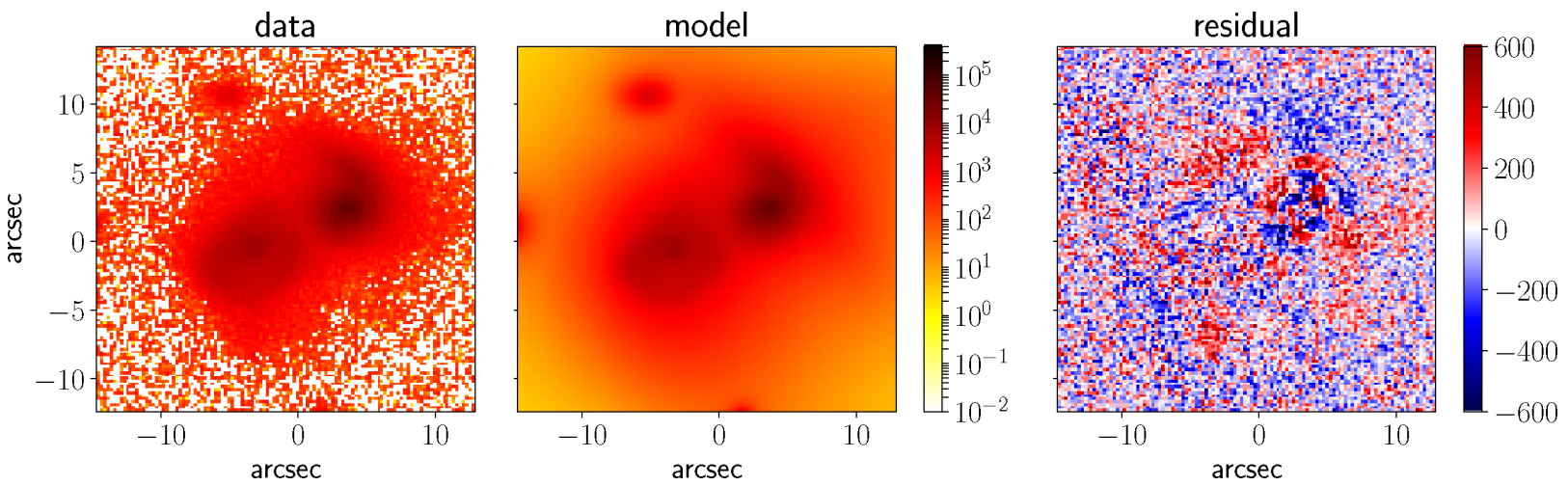

Figure 5. Data, model, and residual in counts, as obtained by fitting the PanSTARRS $i$-band image with GALFIT. The same colour bar is adopted for the panels showing the data and the model.

Table 4. Stellar masses. (1) Source ID; (2) distance in Mpc, as inferred in Section 4.1; (3) absolute $i$-band magnitude; $(4,5) r$-and $i$-band magnitude; (6) logarithm of the galaxies stellar mass.

\begin{tabular}{lccccc}
$\begin{array}{l}\text { Source } \\
(1)\end{array}$ & $D$ & $M_{i}$ & $r$ & $i$ & $\log 10\left(M_{\star} / M_{\odot}\right)$ \\
\hline 1 & $(2)$ & $(3)$ & $(4)$ & $(5)$ & $(6)$ \\
\hline & 291 & -21.2 & & $16.11 \pm 0.01$ & 10.7 \\
2 & 288 & -21.6 & $16.49 \pm 0.02$ & & $15.68 \pm 0.01$ \\
& & & $16.05 \pm 0.02$ & 10.9 \\
\hline
\end{tabular}

fits the extended faint stellar envelope, but also part of the main body of Source 1 .

Uncertainties. Uncertainties on the determination of each mass are dominated by systematics on the distance estimate; however, these are expected to have a similar effect on each mass, not on the mass ratio. For the sake of completeness, statistical uncertainties derived via error propagation assuming the uncertainties indicated in Table 4, and adopting an uncertainty of $1 \mathrm{Mpc}$ on both distances, are $\Delta \log \left(M_{\star} / M_{\odot}\right)=0.03$ for both galaxies, which implies $\Delta M_{1} / M_{2}=0.05$.

\subsection{Star formation rates}

We adopted the relation derived by Hopkins et al. (2003) to estimate star formation rates using the $u$-band magnitudes:

$\mathrm{SFR}_{u}\left(\mathrm{M}_{\odot} \mathrm{yr}^{-1}\right)=\left[\frac{10^{-0.4\left(M_{u}-34.10\right)}}{1.81 \times 10^{21}}\left(\frac{F_{\mathrm{H} \alpha} / F_{\mathrm{H} \beta}}{2.86}\right)^{2.061}\right]^{1.186}$,

where $M_{u}$ is the absolute magnitude derived using Petrosian magnitudes from SDSS-DR13 $\left(m_{u, 1}=18.16\right.$ and $\left.\mathrm{m}_{u, 2}=17.58\right)$, corrected for extinction $\left(A_{u}=0.07\right)$, and assuming the luminosity distances derived in Section 4.1. $F_{\mathrm{H} \alpha}$ and $F_{\mathrm{H} \beta}$ are the stellar absorption corrected emission-line fluxes from Table 3 . We obtained $\mathrm{SFR}_{1} \approx$ $1.5 \mathrm{M}_{\odot} \mathrm{yr}^{-1}$ and $\mathrm{SFR}_{2} \approx 7.2 \mathrm{M}_{\odot} \mathrm{yr}^{-1}$.

With the estimated SFR we evaluated the contribution of highmass X-ray binaries to the X-ray luminosity. We used the relation derived by Grimm, Gilfanov \& Sunyaev 2003:

$$
\begin{aligned}
& L_{2-10 \mathrm{keV}}=2.6 \times 10^{39} \times \mathrm{SFR}^{1.7}\left[\mathrm{M}_{\odot} \mathrm{yr}^{-1}\right], \mathrm{SFR}<5 \mathrm{M}_{\odot} \mathrm{yr}^{-1} \\
& L_{2-10 \mathrm{keV}}=6.7 \times 10^{39} \times \mathrm{SFR}\left[\mathrm{M}_{\odot} \mathrm{yr}^{-1}\right], \mathrm{SFR}>5 \mathrm{M}_{\odot} \mathrm{yr}^{-1},
\end{aligned}
$$

finding that the hard $\mathrm{X}$-ray luminosity due to $\mathrm{X}$-ray binaries amounts to $L_{2-10 \mathrm{keV}, 1} \approx 5_{-3}^{+6} \times 10^{39} \mathrm{erg} \mathrm{s}^{-1}$ and $L_{2-10 \mathrm{keV}, 2} \approx$ $5_{-2}^{+1} \times 10^{40} \mathrm{erg} \mathrm{s}^{-1}$.

\section{DISCUSSION}

For two galaxies to make up a dual AGN system, it is necessary that the galaxies are interacting and that the two nuclei bear the characteristics of 'active nuclei'. We address the presence of these characteristics in the following sections.

\subsection{Evidence for interaction}

Visual inspection of the optical images of the two galaxies (Fig. 1) shows a prominent elongation departing from Source 2 and bending towards Source 1 - possibly a tidal stream. Faint emission is also present in the surroundings of the two galaxies - possibly stars scattered during the gravitational interaction of the two objects. The morphology of the system suggests therefore that the galaxies are indeed interacting.

Optical spectra allowed us to estimate their redshift, which we presented in Table 3. As the gas emission lines can be affected by non-gravitational motions (i.e. inflows and outflows), biasing the redshift estimate, we derived the redshift from the stellar absorption features obtaining $z_{1}=0.0647 \pm 0.0001$ and $z_{2}=0.06417 \pm 0.00003$. The difference between the two redshifts corresponds to a velocity difference $(v=c z)$ of $160 \mathrm{~km} \mathrm{~s}^{-1}$, a value that can be easily accounted for by the peculiar dynamics of two interacting galaxies.

The picture depicted above supports the hypothesis that the galaxies can be considered an interacting system.

We used Ned's Wright cosmology calculator ${ }^{10}$ (Wright 2006) to derive the luminosity distance corresponding to the adopted cosmology (Section 1) and to the redshifts specified above, obtaining the luminosity distances $D_{L, 1} \approx 291 \mathrm{Mpc}$ and $D_{L, 2} \approx 288 \mathrm{Mpc}$. Considering that the angular separation between the nuclei is approximately $8^{\prime \prime}$ or $11 \mathrm{kpc}$, the physical separation between the two galaxies would be approximately $3 \mathrm{Mpc}$ (about four times the separation between the Milky Way and the Andromeda galaxy); however, as noted above, the difference in the measured redshifts could be due to the peculiar dynamics that two interacting galaxies would

\footnotetext{
${ }^{10} \mathrm{http}: / /$ www.astro.ucla.edu/percent7Ewright/CosmoCalc.html
} 

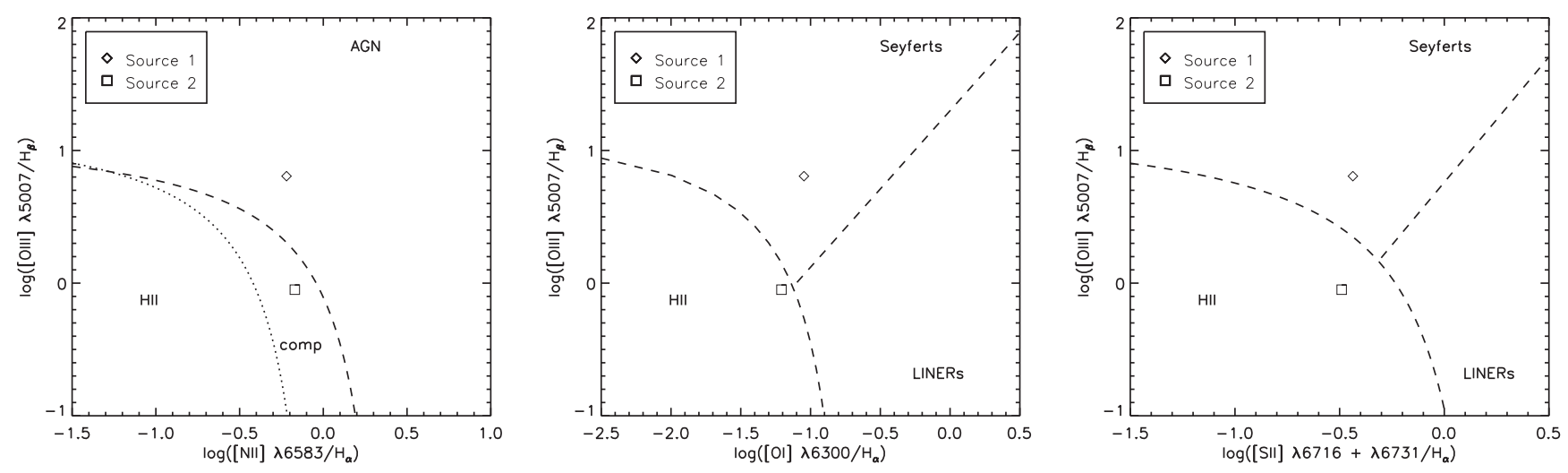

Figure 6. Baldwin-Phillips-Terlevich diagrams for the two galaxies. The dotted and the dashed lines in the left-hand panel mark the empirical and the theoretical maximum starburst lines from Kauffmann et al. (2003) and Kewley et al. (2006), respectively. Error bars are of the order of the symbol size. Clearly, the ratios of the emission lines found in the spectrum of Source 1 are consistent with it harbouring an active SMBH, whereas the conclusion for Source 2 is ambiguous.

experience. Therefore, we believe it is likely that the actual distance between Source 1 and Source 2 is much smaller than $3 \mathrm{Mpc}$.

Uncertainties. The uncertainties on the redshift estimate quoted above is the uncertainty in the result of the fit as measured from Monte Carlo simulation (Section 3.2).

The RMS scatter in the wavelength calibration of the ACAM spectrum $(\delta \lambda=0.5 \AA$, Section 3$)$ corresponds to $\delta z=0.00008$ at $6500 \AA$, or $23 \mathrm{~km} \mathrm{~s}^{-1}$; the uncertainty due to the fitting procedure quoted above is $\delta z=0.0001$ or $\delta v \approx 30 \mathrm{~km} \mathrm{~s}^{-1}$. The resulting statistical uncertainty $\left(\sqrt{\delta z_{1}^{2}+\delta z_{2}^{2}}\right)$ is $\delta z \approx 0.0001$ or $38 \mathrm{~km} \mathrm{~s}^{-1}$. The estimated systematic uncertainty $(\delta \lambda=0.1 \AA$, Section 3$)$ corresponds to $5 \mathrm{~km} \mathrm{~s}^{-1}$.

The uncertainty on the redshift estimated from the SDSS spectrum is dominated by the uncertainty on the wavelength as recovered from the fit, corresponding to $9 \mathrm{~km} \mathrm{~s}^{-1}$.

\subsection{Nuclear activity}

Optical. Emission-line ratios can be used as diagnostics to infer the ionization mechanism. This is of particular interest in our case, as we wish to understand whether AGNs reside in the nuclei of the two galaxies. We plotted the relevant line ratios in BaldwinPhillips-Terlevich diagrams (BPT, Baldwin, Phillips \& Terlevich 1981) adopting the boundaries proposed by Kewley et al. (2006). Results are shown in Fig. 6.

The dominant ionization mechanism in Source 1 is clearly AGN activity. Instead, star formation is the main ionization mechanism in Source 2, which also shows a more uniform blue colour. However, it is worth noting that the $\left[\mathrm{O}_{\mathrm{I}}\right] / \mathrm{H} \alpha$ ratio is close to the boundary between $\mathrm{H}$ II and AGN ionization, and the $[\mathrm{N} \mathrm{II}] / \mathrm{H} \alpha$ line ratio falls in the 'composite' region, which is in between the empirical and theoretical maximum starburst lines. It is therefore possible that a weak AGN resides in Source 2 as well.

Mid-infrared. As shown in Section 3.4, mid-infrared colours derived from WISE photometry (Wright et al. 2010) are characteristics of AGNs for Source 1. The colour of Source 2, instead, falls in between the locus of Starbursts and LIRGs.

When interpreting this result, the reader should note that the WISE PSF has a large full width at half-maximum (between 6 and 7 $\operatorname{arcsec}$ ). The colours are therefore representative of the whole host galaxies, and the contribution of a hypothetical weak AGN residing in Source 2 could easily be overwhelmed by the stellar emission.
$X$-ray. Hard X-ray emission (i.e. at energies in excess of $5 \mathrm{keV}$ ) is an excellent tracer of AGN activity. Such emission is indeed associated with Source 1, which also shows optical line ratios and mid-infrared colours typical of AGN ionization. From the fit of the X-ray spectrum, we derived $L_{x, 2-10 \mathrm{keV}} \approx 5 \times 10^{42} \mathrm{erg} \mathrm{s}^{-1}$ and from the SFR we estimated that the X-ray contribution from $\mathrm{X}$ ray binaries is two-to-three orders of magnitudes lower than the observed values. These numbers firmly identify Source 1 as an active galaxy.

On the other hand, we detected no emission above $2 \mathrm{keV}$ in Source 2. The luminosity extrapolated from the fit in the energy range $2-10 \mathrm{keV}$ is $L_{x}=1.7_{-1.2}^{+1.3} \times 10^{40} \mathrm{erg} \mathrm{s}^{-1}$. This is consistent with the value expected from the observed SFR $\left(5_{-2}^{+1} \times 10^{40} \mathrm{erg} \mathrm{s}^{-1}\right)$; however, the predicted X-ray luminosity derived using the scaling relation of Grimm et al. (2003) refers to the luminosity of the galaxy as a whole. Instead, the X-ray luminosity quoted for Source 2 was derived from a region including no more than about 50 per cent of the galaxy mass (this is approximately the mass ratio between the boxy-bulge and the tadpole body highlighted in the SDSS-band image, Fig. 1). It might be an indication that a weak AGN is present in Source 2.

To frame these results in a broader context, the reader should recall that X-ray emission due to star formation is known to be as high as a few $10^{41} \mathrm{erg} \mathrm{s}^{-1}$ (e.g. Zezas, Alonso-Herrero \& Ward 2001); ultra-luminous X-ray sources have typical luminosities in the range $10^{39}-10^{40} \mathrm{erg} \mathrm{s}^{-1}$; and hyperluminous X-ray sources are known to reach $10^{42} \mathrm{erg} \mathrm{s}^{-1}$ (e.g. Farrell et al. 2009; Heida, Jonker \& Torres 2015).

As in Liu et al. (2013) and Shangguan et al. (2016), we find hard $\mathrm{X}$-ray emission in the nucleus displaying redder colours.

To summarize, while the active nature of Source 1 can be established beyond reasonable doubt, there is only tentative evidence for an AGN in Source 2.

\subsubsection{A note on nuclear point sources}

As stated in Section 3.5, an NPS was used to fit the optical 2D brightness profile of Source 2, while a model without an NPS was adopted for Source 1. The latter can also be modelled with the inclusion of a faint NPS of magnitude $i=20.6$; however, the model does not show any significant improvement, as the reduced chi square remains unaltered and residuals show very little difference. 
The parameters for the resulting nuclear Sérsic component also show minor changes and are mostly consistent with the parameters obtained without an NPS. For these reasons, we adopt the simplest model, which does not include an NPS.

The absence of a bright NPS in Source 1 can be explained noting that Source 1 is host to a Type 2 AGN (lacking permitted broad emission lines in the optical spectrum); therefore, according to the unified model of AGNs (e.g. Urry \& Padovani 1995), a direct view of the SMBH accretion disc (physical counterpart for the NPS) is prevented because of dust obscuration, which we estimated to be relatively high in Source $1\left(n_{\mathrm{H}} \approx 5 \times 22 \mathrm{~cm}^{-2}\right.$, Section 3.1).

On the other hand, the NPS in Source 2, a galaxy dominated by star formation and characterized by a lower nuclear obscuration $\left(n_{\mathrm{H}}\right.$ $\approx 2 \times 10^{20} \mathrm{~cm}^{-2}$ ), could be ascribed to a nuclear star cluster.

\subsection{X-ray versus [OIII] luminosity}

The flux of the $[\mathrm{O}$ III $] \lambda 5007$ emission line originates at scales of hundreds to thousands of parsecs from the ionizing AGN, and it is therefore free of the effects of the nuclear absorption acting on the $\mathrm{X}$-ray emission. Extinction due to the large-scale dust distribution can be inferred from the Balmer decrement. As a result, the $[\mathrm{O}$ III] luminosity has been proposed as a proxy for the AGN true luminosity, and it has been used to infer the X-ray absorption (e.g. Bassani et al. 1999).

Liu et al. (2013) showed tentative evidence that optically selected kpc-scale dual AGNs do not follow the trend defined by isolated AGNs, displaying a lower X-ray-to-[O III] ratio; this could be an indication that large amounts of gas and dust are funnelled towards the centre during the merger process.

We derived the extinction-corrected [O III] luminosity as

$L_{\text {[O III] }}^{c}=L_{\text {[O III] }}\left(\frac{F_{\mathrm{H} \alpha} / F_{\mathrm{H} \beta}}{3}\right)^{2.94}$,

where $L_{[\mathrm{O} \mathrm{III}]}$ is the observed luminosity of the [O III] emission line as derived from the fluxes in Table 3 (respectively $L_{[\mathrm{O} \text { II] }], 1}=1.46 \times 10^{41} \mathrm{erg} \mathrm{s}^{-1}$ and $L_{[\mathrm{O} \mathrm{II}], 2}=4.94 \times 10^{40} \mathrm{erg} \mathrm{s}^{-1}$ ), $F_{\mathrm{H} \alpha}$ and $F_{\mathrm{H} \beta}$ are the observed fluxes of the $\mathrm{H} \alpha$ and $\mathrm{H} \beta$ emission lines, and an intrinsic Balmer decrement of 3.0 was assumed (Osterbrock \& Ferland 2006). This resulted into $L_{[\mathrm{O} \amalg], 1}^{c} \approx$ $2 \times 10^{41} \mathrm{erg} \mathrm{s}^{-1}$ and $L_{[\mathrm{O} \text { II] }], 2}^{c} \approx 2 \times 10^{39} \mathrm{erg} \mathrm{s}^{-1}$.

The location of our targets in an $L_{x, 2-10 \mathrm{keV}}$ versus $L_{[\mathrm{O} \text { II] }}$ is shown in Fig. 7, along with a comparison sample of Seyferts and LINERS drawn from the Palomar survey of nearby galaxies (Ho, Filippenko $\&$ Sargent 1995) by Panessa et al. (2006): the two nuclei fall within the locus defined by single AGNs. With the caveat that the $L_{x, 2-10 \mathrm{keV}}$ for Source 2 is an extrapolation based on the emission at energies below $2 \mathrm{keV}$, the result might be an indication that Source 2 does harbour a low-luminosity AGN.

\subsection{Eddington ratio}

To estimate the Eddington ratio of Source 1 (the galaxy with strong evidence for AGN activity), $\lambda_{E}=L_{\mathrm{bol}} / L_{\mathrm{Edd}}$, we need to estimate the bolometric luminosity $\left(L_{\mathrm{bol}}\right)$ and the Eddington luminosity $\left(L_{\mathrm{Edd}}\right)$.

We computed the bolometric luminosity using scaling relations with the optical and X-ray data. In the optical, we adopted the bolometric correction $\left(C_{[\mathrm{O} \text { III }]}=L_{\text {bol }} / L_{[\mathrm{O} \text { III }]}^{c}\right)$ derived by Lamastra et al. (2009) from extinction-corrected [O III] emission lines. In Section 4.3, we derived $L_{[\mathrm{O} \text { ㅍ] }, 1}^{c} \approx 2 \times 10^{41} \mathrm{erg} \mathrm{s}^{-1}$, which requires a bolometric correction $C_{[\mathrm{O} \mathrm{II}]}=87$, producing $L_{\mathrm{bol}, 1} \approx$ $1 \times 10^{43} \mathrm{erg} \mathrm{s}^{-1}$.
We also estimated the bolometric luminosity using the scaling relation of Ho (2009): $C_{X}=L_{\mathrm{bol}} / L_{X, 2-10 \mathrm{keV}} \approx 8$, which produces $L_{\mathrm{bol}, 1} \approx 2 \times 10^{43} \mathrm{erg} \mathrm{s}^{-1}$. However, Ho argued that a larger data set supports $C_{X} \approx 15.8$. Given this uncertainty, and the consistency between the different estimates, we adopted the value derived from the $[\mathrm{O}$ III] emission line.

The Eddington luminosity is defined as

$L_{E}=4 \pi c G M_{\bullet} m_{\mathrm{p}} / \sigma_{T}$,

where $c$ is the speed of light, $m_{\mathrm{p}}$ the mass of the proton, $\sigma_{T}$ the Thomson cross-section, and $M_{\bullet}$ is the mass of the $\mathrm{BH}$, which is responsible for the nuclear activity.

The mass of the SMBH is very uncertain; nevertheless, we present an estimate based on two approaches: the scaling relation between SMBH mass and bulge Sérsic index (Savorgnan et al. 2013), and the scaling relation between SMBH mass and bulge velocity dispersion (Ferrarese \& Merritt 2000; Gebhardt et al. 2000).

To warrant the use of such scaling relations, one should find evidence for a bulge. The task is not trivial and multiple lines of evidence should be put forward (such as photometric, possibly from high-resolution images, and kinematical, e.g. Gadotti 2012). Our claim for the presence of a bulge-like component in Source 1 is based on the 2D decomposition of the $i$-band image presented in Section 3.5, where we showed that a satisfactory fit can be achieved with the inclusion of a compact Sérsic component with low index (\#1 in Fig. A2), possibly a rotationally supported bulge.

$M_{\bullet}$ - Sérsic index: For Source 1, we measured the Sérsic index $n_{1}=0.3 \pm 0.1$. Using the scaling relation presented in Savorgnan et al. (2013),

$\log \left(M_{\bullet}\right)=(7.85 \pm 0.14)+(3.38 \pm 1.16) \log (n / 3)$,

we obtained $M_{\bullet} \sim 10^{4} \mathrm{M}_{\odot}$, which corresponds to the Eddington luminosity $L_{E} \sim 10^{42} \mathrm{erg} \mathrm{s}^{-1}$. The resulting Eddington ratio is $\lambda_{E} \approx$ 4.

Caveats and uncertainties. The $M_{\bullet}-n$ relation was derived using Sérsic indices $n \geq 1$; the correlation, with a scatter of $0.44 \mathrm{dex}$, is therefore unconstrained for $n<1$.

The structural parameters derived in a multicomponent fit could be affected by degeneracies (see the detailed discussion in Peng et al. 2010) and the Sérsic index is also affected by orientation and extinction (Pastrav et al. 2013).

Removing or adding azimuthal shape functions (i.e. Fourier modes and bending modes) in the components that we adopted in our GALFIT model caused variations in the Sérsic index, which were never larger than 9 percent. We observed similar variations when varying the initial guess. However, the removal of a low-luminosity component, such as the exponential disc used to model the irregularities of Source 1 and the stellar envelope, caused a variation as large as 20 percent. This is the value that we assumed to represent the systematic errors in the Sérsic index, which corresponds to a systematic uncertainty on the Eddington ratio $\lambda_{E, 1} \approx 4_{-2}^{+5}$.

Systematics in the Sérsic index due, separately, to the orientation and the dust, as derived from bulge-disc ( $\mathrm{B} / \mathrm{D})$ decomposition of model galaxies, are always in the range $-0.1<n^{\mathrm{B} / \mathrm{D}}-n^{\text {single }}<+0.3$, with the correction being between -0.05 and +0.05 for intermediate inclinations, where $n^{\mathrm{B} / \mathrm{D}}$ and $n^{\text {single }}$ are the Sérsic indices of decomposed and single bulges, respectively (Pastrav et al. 2013). These uncertainties, at intermediate inclinations, correspond to a systematic error in $\lambda_{E, 1}$ of $-2,+3$ due to the effect of dust, and $-2,+3$ due to effect of orientation.

$M_{\bullet}$ - stellar velocity dispersion: The observed stellar velocity dispersion for Source 1 is $\sigma_{\mathrm{obs}}=284 \pm 38 \mathrm{~km} \mathrm{~s}^{-1}$. Given that 

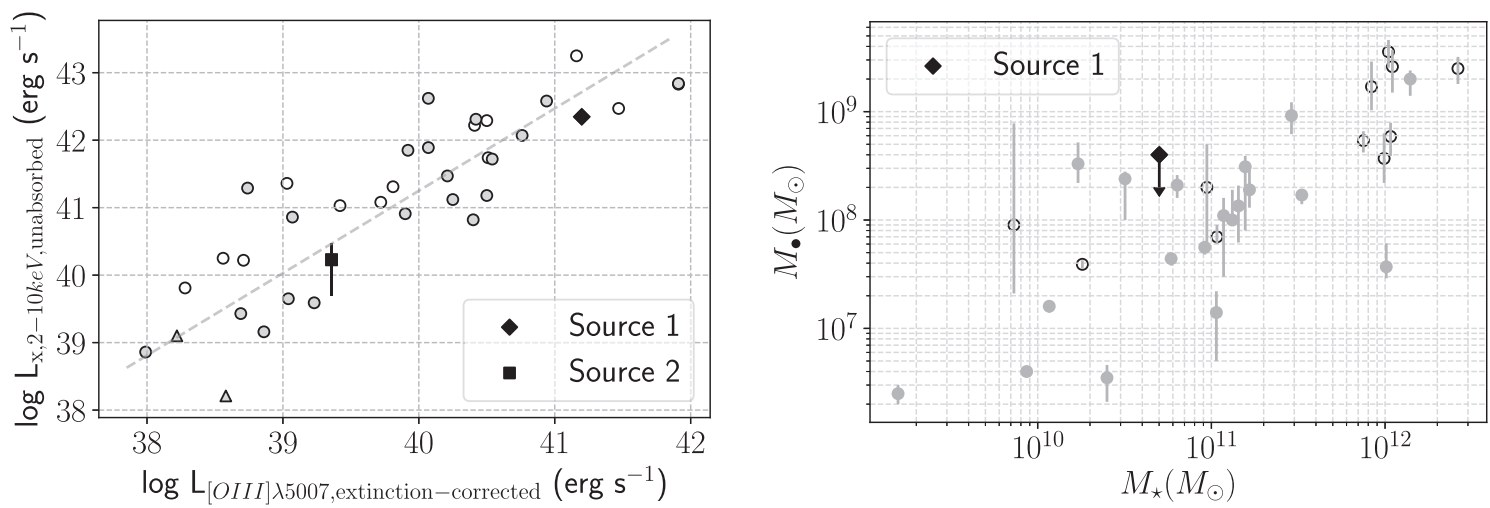

Figure 7. Left: Unabsorbed $2-10 \mathrm{keV}$ luminosity versus extinction-corrected [OIII] luminosity. The comparison sample is taken from Panessa et al. (2006) and it includes galaxies classified as Seyfert 1-1.5 (open circles), Seyferts 1.8-2 (grey circles), and two galaxies classified as LINER2/Sy2 (grey triangles). Galaxies for which the classification was marked as uncertain or highly uncertain were not included into this plot. The dashed line is the correlation derived by Panessa et al. for the mixed sample of Seyferts [i.e. $\left.\log _{x}=(1.22 \pm 0.11) \times \log _{[\mathrm{O}} \mathrm{II]}-(7.55 \pm 4.33)\right]$. When omitted, error bars for Source 1 and 2 are smaller than the symbol size. Note that the X-ray luminosity for Source 2 is an extrapolation from the fit. Right: BH mass versus stellar galaxy mass. The comparison sample consists of galaxies from table II in Ferrarese \& Ford (2005, excluding galaxies for which dynamical models are deemed to be in error). BH masses are taken from Ferrarese \& Ford (2005, see references therein); virial stellar masses have been estimated as $\alpha R_{\mathrm{e}} \sigma^{2} / G$, with $\alpha=5$ (Cappellari et al. 2006), $R_{\mathrm{e}}$, the effective radius, and $\sigma$ the stellar velocity dispersion from Marconi \& Hunt (2003). Grey dots: non-active galaxies; black circles: active galaxies (as classified on the NASA/IPAC Extragalactic Database).

the stellar velocity dispersion is derived from spectral features distributed over the full spectral range (5000-7500 $\AA$ ), we corrected this value for the instrumental resolution $\sigma_{\text {ins }}=271 \mathrm{~km} \mathrm{~s}^{-1}$, which is the theoretical instrumental velocity dispersion at $6150 \AA$, assuming the nominal dispersion of $13.1 \AA^{11} .^{11}$ The resulting intrinsic stellar velocity dispersion $\left(\sqrt{\sigma_{\text {obs }}^{2}-\sigma_{\text {ins }}^{2}}\right)$ is $\sigma=84_{-84}^{+127} \mathrm{~km} \mathrm{~s}^{-1}$, where we estimated the uncertainty using the error propagation formula for independent variables, assuming no uncertainty on $\sigma_{\text {ins }}$. This value can be used in conjunction with the $M_{\bullet}-\sigma$ relation presented in Ferrarese \& Ford (2005):

$$
\frac{M_{\bullet}}{10^{8} \mathrm{M}_{\odot}}=(1.66 \pm 0.24)\left(\frac{\sigma}{200 \mathrm{kms}^{-1}}\right)^{4.86 \pm 0.43} .
$$

As in Ferrarese \& Merritt (2000), we applied an aperture correction to the velocity dispersion normalizing it to an aperture of radius $1 / 8$ of the bulge effective radius. Towards this end, we used the prescription of Jorgensen, Franx \& Kjaergaard (1995):

$\sigma_{\mathrm{e} 8}=\sigma_{\mathrm{ap}}\left(\frac{r_{\mathrm{e}} / 8}{r_{\mathrm{ap}}}\right)^{-0.04}$

where $r_{\mathrm{e}}=0.6$ arcsec is the bulge effective radius as derived from GALFIT (taking into account the PanSTARRS pixel size of $0.258 \mathrm{arcsec}$ ), $r_{\mathrm{ap}}=1.75 \mathrm{arcsec}$ is the radius of the aperture used in IRAF to extract the spectrum, and $\sigma_{\text {ap }}=84 \mathrm{~km} \mathrm{~s}^{-1}$ is the observed stellar velocity dispersion corrected for instrumental broadening. We obtained $\sigma_{8}=95_{-95}^{+144} \mathrm{~km} \mathrm{~s}^{-1}$.

Given the large uncertainty on the stellar velocity dispersion, we estimated an upper limit to the $\mathrm{SMBH}$ mass: adopting $\sigma$ $\leq 239 \mathrm{~km} \mathrm{~s}^{-1}$, we obtained $M_{\bullet} \leq 4 \times 10^{8} \mathrm{M}_{\odot}$ and $\mathrm{L}_{E} \leq$ $5 \times 10^{46} \mathrm{erg} \mathrm{s}^{-1}$. The resulting Eddington ratio is $\lambda_{E} \geq 10^{-4}$.

When compared to other BHs residing in galaxies of similar masses, we can only conclude that the BH in Source 1 is not obviously overmassive (Fig. 7).

\footnotetext{
${ }^{11} \sigma_{\text {ins }}=\frac{c}{2.355} \frac{\Delta \lambda}{\lambda}$, with $c$ the speed of light and $\Delta \lambda=13.1 \AA$ the instrumental resolution. The high value of $\sigma_{\text {ins }}$, that is the low resolution of ACAM, is responsible for the large uncertainty on the 'intrinsic' stellar velocity dispersion, which appears later in the text.
}

To summarize, because of the large uncertainties on the SMBH mass, the Eddington ratio is poorly constrained, with $10^{-4} \leq \lambda_{E} \leq$ 9.

\section{SUMMARY AND CONCLUSIONS}

In SDSS-DR7, we selected galaxies separated by no more than 10 times the sum of their Petrosian radii. Subsequently, we crosscorrelated the sample with the $X M M$ serendipitous source catalogue (2XMMi) looking for galaxy pairs associated with a single X-ray source (possibly a recoiling SMBH). The search produced two candidates: the hyperluminous X-ray source described in Jonker et al. (2010) and the system studied in this paper: a pair of interacting galaxies with stellar mass ratio $q \approx 0.7$ and an X-ray source approximately coincident with the optical nucleus of the Eastern galaxy (Source 1). To further investigate the nature of the system, we performed Chandra observations, which revealed a nuclear Xray source in the Western galaxy as well (Source 2), and we used ACAM on the William Herschel Telescope to obtain a new optical spectrum for Source 1.

Source 1 shows hard X-ray emission, low star formation rate ( $2 \mathrm{M}_{\odot} \mathrm{yr}^{-1}$ ), a redder nucleus with mid-infrared colours located within the locus of AGN candidates, and optical emission-line ratios typical of AGN photoionization. The active nature of this galaxy is evident.

Source 2, instead, has a softer spectrum with no emission above $2 \mathrm{keV}$; it has a higher star formation rate $\left(7 \mathrm{M}_{\odot} \mathrm{yr}^{-1}\right)$; indeed, midinfrared colours are typical of Starburst/LINERs; the optical emissionline ratios are also characteristics of $\mathrm{H}$ II regions. However, they are close to the demarcation between starburst- and AGN-driven ionization, with the $\left[\mathrm{N}_{\mathrm{II}}\right] / \mathrm{H} \alpha$ emission-line ratio falling in the 'composite' region.

When plotted on an $L_{x, 2-10 \mathrm{keV}}$ versus $L_{[\mathrm{O} \text { III] }}$ diagram, both nuclei fall within the locus defined by a mixed sample of local AGNs, mostly Seyfert galaxies. Moreover, the $2-10 \mathrm{keV}$ luminosity extracted from the inner region of Source 2 is consistent with the value expected for the galaxy as a whole on the basis of the star formation rate. With the caveat that the hard X-ray luminosity for 
Source 2 is an extrapolation based on the soft X-ray spectrum, these are, perhaps, hints that a low-luminosity AGN is buried within Source 2.

Using the relative intensity of the $[\mathrm{S}$ II] emission lines, we estimated the electron densities finding that Source 1 has a density $n_{\mathrm{e}}<27 \mathrm{e}^{-} \mathrm{cm}^{-3}$, while Source 2 has an electron density of approximately $200 \mathrm{e}^{-} \mathrm{cm}^{-3}$.

We used GALFIT to perform a 2D decomposition of the light profiles. Both galaxies can be modelled with an inner Sérsic component with an index $n<1$, suggesting the presence of rotationally supported bulges. For Source 1, we estimated the Eddington ratio, $\lambda_{E}$; however, the $\mathrm{BH}$ mass, estimated using two scaling relations $\left(M_{\bullet}\right.$ versus Sérsic index, and $M_{\bullet}$ versus stellar velocity dispersion), is highly uncertain leaving $\lambda_{E}$ essentially unconstrained.

While the active nature of Source 1 can be established with confidence, our analysis does not allow a definitive statement about the presence of an AGN within Source 2. However, hints of its existence are tantalizing. Bearing in mind the importance of defining a well-characterized, secure sample of AGNs within interacting galaxies, we will further probe the nature of Source 2 with dualfrequency radio observations. The detection of a compact steepspectrum radio source would definitely prove the active nature of its nucleus.

\section{ACKNOWLEDGEMENTS}

The authors thank K. López-López for carrying out the ACAM observations and the anonymous referee for valuable comments and suggestions. DL and PGJ acknowledge support from the European Research Council (ERC) under grant 647208 (PI Jonker). DL thanks the organizers and the participants of the Lorentz Center workshop 'The quest for multiple supermassive black holes: a multi-messenger view' for constructive and informative discussions; DL also thanks D. Merritt for constructive comments and C. Y. Peng and M. Cappellari for timely and clear assistance with the use of GALFIT and PPXF, respectively. MAPT acknowledges support via a Ramn y Cajal Fellowship (RYC-2015-17854).

This research is based on observations made with the William Herschel Telescope, operated on the island of La Palma by the Isaac Newton Group of Telescopes in the Spanish Observatorio del Roque de los Muchachos of the Instituto de Astrofísica de Canarias. This research has made use of the NASA/IPAC Extragalactic Database (NED), which is operated by the Jet Propulsion Laboratory, California Institute of Technology, under contract with the National Aeronautics and Space Administration. This reasearch has also made use of data products from the Wide field Infrared Survey Explorer, which is a joint project of the University of California, Los Angeles, and the Jet Propulsion Laboratory/California Institute of Technology, funded by the National Aeronautics and Space Administration.

\section{REFERENCES}

Abazajian K. N. et al., 2009, ApJS, 182, 543

Albareti F. D. et al., 2017, ApJS, 233, 25

Alexander D. M., Hickox R. C., 2012, New A Rev., 56, 93

Arnaud K. A., 1996, in Jacoby G. H., Barnes J., eds, ASP Conf. Ser. Vol. 101, Astronomical Data Analysis Software and Systems V. Astron. Soc. Pac., San Francisco, p. 17

Baldwin J. A., Phillips M. M., Terlevich R., 1981, PASP, 93, 5

Barrows R. S., Sandberg Lacy C. H., Kennefick J., Comerford J. M., Kennefick D., Berrier J. C., 2013, ApJ, 769, 95
Bassani L., Dadina M., Maiolino R., Salvati M., Risaliti G., Della Ceca R., Matt G., Zamorani G., 1999, ApJS, 121, 473

Bekenstein J. D., 1973, ApJ, 183, 657

Bell E. F., McIntosh D. H., Katz N., Weinberg M. D., 2003, ApJS, 149, 289

Benn C., Dee K., Agócs T., 2008, in McLean I. S., Casali M. M., eds, Proc. SPIE Conf. Ser., Vol. 7014, Ground-based and Airborne Instrumentation for Astronomy II. SPIE, Bellingham, p. 70146X

Bennert N., Jungwiert B., Komossa S., Haas M., Chini R., 2006, A\&A, 456, 953

Blanton M. R., Roweis S., 2007, AJ, 133, 734

Blecha L., Loeb A., Narayan R., 2013, MNRAS, 429, 2594

Brum C., Riffel R. A., Storchi-Bergmann T., Robinson A., Schnorr Müller A., Lena D., 2017, MNRAS, 469, 3405

Buta R., Combes F., 1996, Fundam. Cosm. Phys., 17, 95

Callegari S., Kazantzidis S., Mayer L., Colpi M., Bellovary J. M., Quinn T., Wadsley J., 2011, ApJ, 729, 85

Campanelli M., Lousto C., Zlochower Y., Merritt D., 2007, ApJ, 659, L5

Cappellari M., 2017, MNRAS, 466, 798

Cappellari M., Emsellem E., 2004, PASP, 116, 138

Cappellari M. et al., 2006, MNRAS, 366, 1126

Cash W., 1979, ApJ, 228, 939

Chambers K. C. et al., 2016, preprint (arXiv:1612.05560)

Civano F. et al., 2010, ApJ, 717, 209

Civano F. et al., 2012, ApJ, 752, 49

Comerford J. M., Gerke B. F., Stern D., Cooper M. C., Weiner B. J., Newman J. A., Madsen K., Barrows R. S., 2012, ApJ, 753, 42

Crenshaw D. M., Kraemer S. B., George I. M., 2003, ARA\&A, 41, 117

Crenshaw D. M., Schmitt H. R., Kraemer S. B., Mushotzky R. F., Dunn J. P., 2010, ApJ, 708, 419

Davies R. L. et al., 2017, MNRAS, 470, 4974

De Robertis M. M., Dufour R. J., Hunt R. W., 1987, J. R. Astron. Soc. Can., 81,195

Dotti M., Merloni A., Montuori C., 2015, MNRAS, 448, 3603

Emsellem E., Renaud F., Bournaud F., Elmegreen B., Combes F., Gabor J. M., 2015, MNRAS, 446, 2468

Escala A., Becerra F., del Valle L., Castillo E., 2013, ApJ, 763, 39

Farrell S. A., Webb N. A., Barret D., Godet O., Rodrigues J. M., 2009, Nature, 460, 73

Ferrarese L., Ford H., 2005, Space Sci. Rev., 116, 523

Ferrarese L., Merritt D., 2000, ApJ, 539, L9

Freeman P. E., Kashyap V., Rosner R., Lamb D. Q., 2002, ApJS, 138, 185

Fruscione A. et al., 2006, in Silva D. R., Doxsey R. E., eds, Proc. SPIE Conf. Ser. Vol. 6270, Observatory Operations: Strategies, Processes, and Systems. SPIE, Bellingham, p. 62701V

Fu H. et al., 2011, ApJ, 740, L44

Gabányi K. É., An T., Frey S., Komossa S., Paragi Z., Hong X.-Y., Shen Z.-Q., 2016, ApJ, 826, 106

Gabányi K. É., Frey S., Paragi Z., An T., Komossa S., 2017, in Gomboc A., ed., Proc. IAU Symp. 324, New Frontiers in Black Hole Astrophysics. Cambdridge Univ. Press, Cambridge, p. 223

Gadotti D. A., 2012, preprint (arXiv:1208.2295)

Gaia Collaboration, 2016, A\&A, 595, A2

Garmire G. P., Bautz M. W., Ford P. G., Nousek J. A., Ricker G. R., Jr, 2003, in Truemper J. E., Tananbaum H. D., eds, Proc. SPIE Vol. 4851, X-Ray and Gamma-Ray Telescopes and Instruments for Astronomy. SPIE, Bellingham, p. 28

Gebhardt K. et al., 2000, ApJ, 539, L13

Grimm H.-J., Gilfanov M., Sunyaev R., 2003, Chin. J. Astron. Astrophys. Suppl., 3, 257

Haiman Z., 2017, Phys. Rev. D, 96, 023004

Hainline K. N., Hickox R. C., Chen C.-T., Carroll C. M., Jones M. L., Zervos A. S., Goulding A. D., 2016, ApJ, 823, 42

Heckman T. M., Best P. N., 2014, ARA\&A, 52, 589

Heckman T. M., Miley G. K., Green R. F., 1984, ApJ, 281, 525

Heida M., Jonker P. G., Torres M. A. P., 2015, MNRAS, 454, L26

Hickox R. C., Mullaney J. R., Alexander D. M., Chen C.-T. J., Civano F. M., Goulding A. D., Hainline K. N., 2014, ApJ, 782, 9 
Ho L. C., 1996, in Eracleous M., Koratkar A., Leitherer C., Ho L., eds, ASP Conf. Ser. Vol. 103, The Physics of Liners in View of Recent Observations. Astron. Soc. Pac., San Francisco, p. 103

Ho L. C., 2009, ApJ, 699, 626

Ho L. C., Filippenko A. V., Sargent W. L., 1995, ApJS, 98, 477

Hopkins P. F., Quataert E., 2010, MNRAS, 407, 1529

Hopkins A. M. et al., 2003, ApJ, 599, 971

Hopkins P. F., Somerville R. S., Hernquist L., Cox T. J., Robertson B., Li Y., 2006, ApJ, 652, 864

Jonker P. G., Torres M. A. P., Fabian A. C., Heida M., Miniutti G., Pooley D., 2010, MNRAS, 407, 645

Jorgensen I., Franx M., Kjaergaard P., 1995, MNRAS, 276, 1341

Kalberla P. M. W., Burton W. B., Hartmann D., Arnal E. M., Bajaja E., Morras R., Pöppel W. G. L., 2005, A\&A, 440, 775

Kauffmann G. et al., 2003, MNRAS, 346, 1055

Kewley L. J., Groves B., Kauffmann G., Heckman T., 2006, MNRAS, 372, 961

Khan F. M., Fiacconi D., Mayer L., Berczik P., Just A., 2016, ApJ, 828, 73

Komossa S., Burwitz V., Hasinger G., Predehl P., Kaastra J. S., Ikebe Y., 2003, ApJ, 582, L15

Komossa S., Zhou H., Lu H., 2008, ApJ, 678, L81

Koss M. et al., 2011a, ApJ, 735, L42

Koss M., Mushotzky R., Veilleux S., Winter L. M., Baumgartner W., Tueller J., Gehrels N., Valencic L., 2011b, ApJ, 739, 57

Koss M., Mushotzky R., Treister E., Veilleux S., Vasudevan R., Trippe M., 2012, ApJ, 746, L22

Kron R. G., 1980, ApJS, 43, 305

Lamastra A., Bianchi S., Matt G., Perola G. C., Barcons X., Carrera F. J., 2009, A\&A, 504, 73

Lena D., Robinson A., Marconi A., Axon D. J., Capetti A., Merritt D., Batcheldor D., 2014, ApJ, 795, 146

Lena D. et al., 2015, ApJ, 806, 84

Lena D., Robinson A., Storchi-Bergmann T., Couto G. S., Schnorr-Müller A., Riffel R. A., 2016, MNRAS, 459, 4485

Li J., Kastner J. H., Prigozhin G. Y., Schulz N. S., Feigelson E. D., Getman K. V., 2004, ApJ, 610, 1204

Liu X., Shen Y., Strauss M. A., Hao L., 2011, ApJ, 737, 101

Liu X., Civano F., Shen Y., Green P., Greene J. E., Strauss M. A., 2013, ApJ, 762,110

Lousto C. O., Zlochower Y., 2013, Phys. Rev. D, 87, 084027

Marconi A., Hunt L. K., 2003, ApJ, 589, L21

Massaro F., D’Abrusco R., Ajello M., Grindlay J. E., Smith H. A., 2011, ApJ, 740, L48

Mazzarella J. M. et al., 2012, AJ, 144, 125

McGurk R. C., Max C. E., Medling A. M., Shields G. A., Comerford J. M., 2015, ApJ, 811, 14

Merritt D., 2013, Dynamics and Evolution of Galactic Nuclei. Princeton Univ. Press, Princeton

Merritt D., Ferrarese L., 2001, MNRAS, 320, L30

Milosavljević M., Phinney E. S., 2005, ApJ, 622, L93

Müller-Sánchez F., Comerford J., Stern D., Harrison F. A., 2016, ApJ, 830 , 50

Nevin R., Comerford J. M., Müller-Sánchez F., Barrows R., Cooper M. C., 2018, MNRAS, 473, 2160
Osterbrock D. E., Ferland G. J., 2006, Astrophysics of Gaseous Nebulae and Active Galactic Nuclei, 2nd edn. University Science Books, Sausalito, CA

Panessa F., Bassani L., Cappi M., Dadina M., Barcons X., Carrera F. J., Ho L. C., Iwasawa K., 2006, A\&A, 455, 173

Pastrav B. A., Popescu C. C., Tuffs R. J., Sansom A. E., 2013, A\&A, 557, A137

Peng C. Y., Ho L. C., Impey C. D., Rix H.-W., 2002, AJ, 124, 266

Peng C. Y., Ho L. C., Impey C. D., Rix H.-W., 2010, AJ, 139, 2097

Pérez-Ramírez D., Knapen J. H., Peletier R. F., Laine S., Doyon R., Nadeau D., 2000, MNRAS, 317, 234

Perryman M. A. C. et al., 2001, A\&A, 369, 339

Petrosian V., 1976, ApJ, 209, L1

Risaliti G., Elvis M., 2004, in Barger A. J., ed., Astrophysics and Space Science Library, Vol. 308, Supermassive Black Holes in the Distant Universe. Kluwer, Dordrecht, p. 187

Sánchez-Blázquez P. et al., 2006, MNRAS, 371, 703

Sanders R. L. et al., 2016, ApJ, 816, 23

Satyapal S. et al., 2017, ApJ, 848, 126

Savorgnan G., Graham A. W., Marconi A., Sani E., Hunt L. K., Vika M., Driver S. P., 2013, MNRAS, 434, 387

Schawinski K., Koss M., Berney S., Sartori L. F., 2015, MNRAS, 451, 2517

Schnorr Müller A., Storchi-Bergmann T., Riffel R. A., Ferrari F., Steiner J. E., Axon D. J., Robinson A., 2011, MNRAS, 413, 149

Shangguan J., Liu X., Ho L. C., Shen Y., Peng C. Y., Greene J. E., Strauss M. A., 2016, ApJ, 823, 50

Shaw R. A., Dufour R. J., 1995, PASP, 107, 896

Shi Z.-X. et al., 2014, Res. Astron. Astrophys., 14, 1234

Stern D. et al., 2012, ApJ, 753, 30

Tichy W., Marronetti P., 2007, Phys. Rev. D, 76, 061502

Tonry J. L. et al., 2012, ApJ, 750, 99

Urry C. M., Padovani P., 1995, PASP, 107, 803

Van Wassenhove S., Volonteri M., Mayer L., Dotti M., Bellovary J., Callegari S., 2012, ApJ, 748, L7

Wang J.-M., Chen Y.-M., Hu C., Mao W.-M., Zhang S., Bian W.-H., 2009, ApJ, 705, L76

Watson M. G. et al., 2009, A\&A, 493, 339

Weisskopf M. C., Tananbaum H. D., Van Speybroeck L. P., O’Dell S. L., 2000, in Truemper J. E., Aschenbach B., eds, Proc. SPIE Vol. 4012, X-Ray Optics, Instruments, and Missions III. SPIE, Bellingham, p. 2

Wright E. L., 2006, PASP, 118, 1711

Wright E. L. et al., 2010, AJ, 140, 1868

Xu D., Komossa S., 2009, ApJ, 705, L20

Zacharias N., Finch C. T., Girard T. M., Henden A., Bartlett J. L., Monet D. G., Zacharias M. I., 2013, AJ, 145, 44

Zezas A., Alonso-Herrero A., Ward M. J., 2001, Ap\&SS, 276, 601

Zhou H., Wang T., Zhang X., Dong X., Li C., 2004, ApJ, 604, L33

\section{APPENDIX A: GALFIT COMPONENTS}

Best-fitting subcomponents are plotted in Fig. A1; parameters are shown in Fig. A2. 

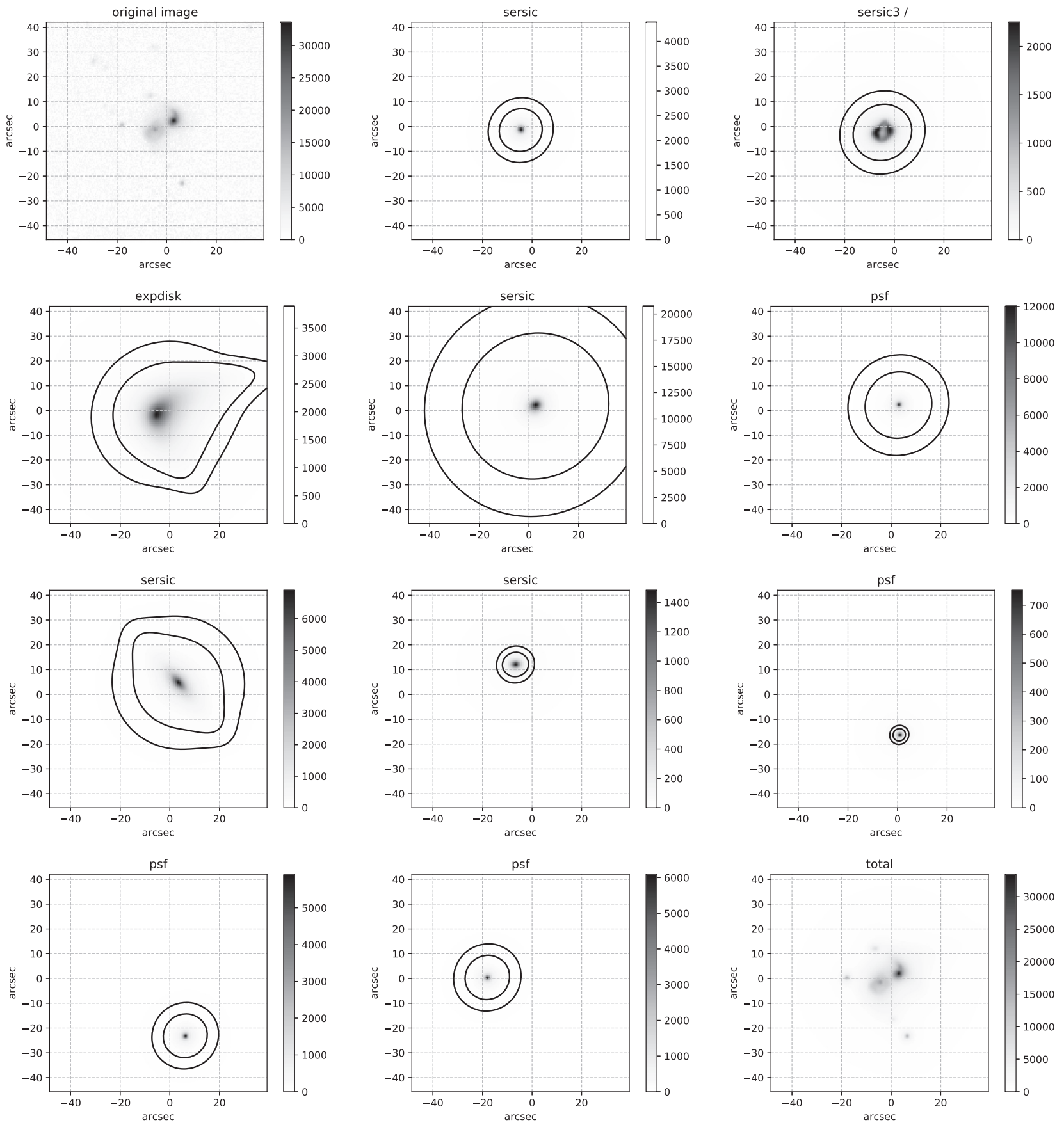

Figure A1. Components fitted via GALFIT to the $i$-band PanSTARRS image. For visualization purpose, we plot the square root of the pixel values. The contours, corresponding to 40 and 80 counts, have been arbitrarily chosen to highlight the low-luminosity extension of the components. See Fig. 5 for a zoom-in on the central region. 


\begin{tabular}{|c|c|c|c|}
\hline & & $1 \mathrm{t}$ & \\
\hline \multicolumn{4}{|c|}{ 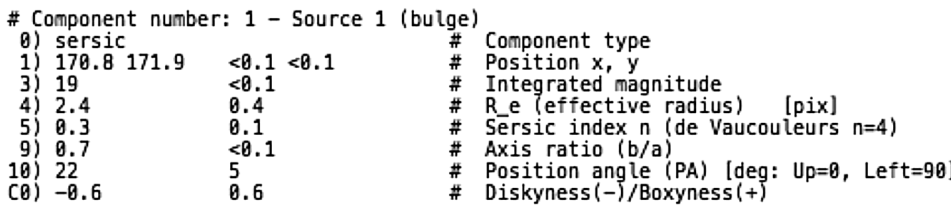 } \\
\hline
\end{tabular}

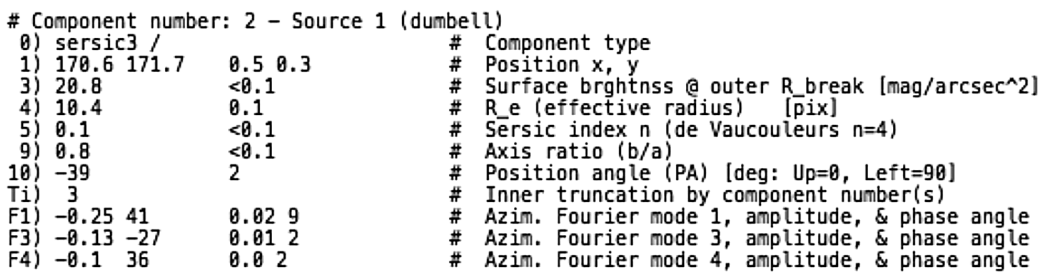

\# Component number: 3 - Source 1 (dumbell)

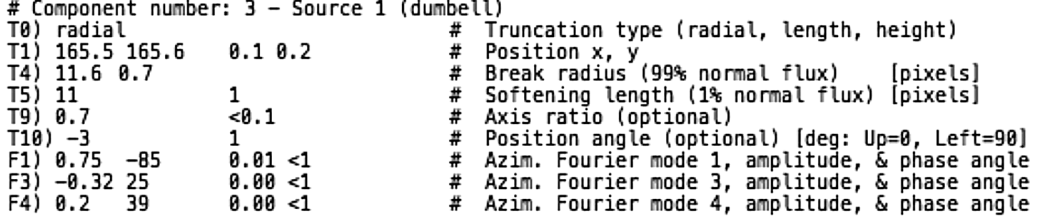

\# Component number: 4 - Source 1 (stellar envelope, and irregularities of Source 1)

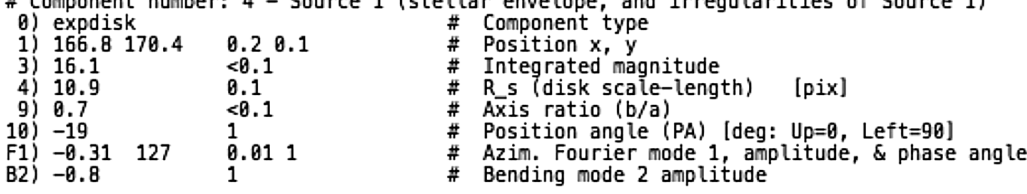

\# Component number: 5 - Source 2 (bulge)
0) sersic
1) 199.2
3) 16.2
4) 5.7
5) 0.7
19) 0.8
F1) $0.19-150$
$<0.1<0.1$
\# Component type
\# Position $x, y$
\# Integrated magnitude
\# Sersic index $n$ (de vaucouleurs $n=4$
$<0.1$
$<0.1 \quad$ \# Sersic index $n$
\# Position angle (PA) [deg: Up=0, Left $=90$ ]

\# Component number: 6 - Source 2 (nuclear point source)
O) psf

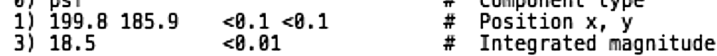

\# Component number: 7 - Source 2 (tail)

0) sersic

1) 201.8195 .9

3) 17

5) 11.6

5) 1.5

$<0.1<0.1$

$<0.1$

0.1

$<0.1$

(0)

\# Component type

10) $4 i^{4}$

$<1$

\# Position typ

\# Integrated magnitude

\# R_e (effective radius) [pix]

\# Sērsic index $n$ (de Vaucouleurs $n=4$ )

\# Axis ratio (b/a)

\# Component number: 8 - nearby galaxy (north)

$\begin{array}{llll}\text { \# Component number: } 8 \text { - nearby galaxy (north) } \\ \text { 0) sersic } & & \text { \# Component type } \\ \text { 1) } 162.6 & 224.2 & 0.10 .1 & \text { \# Position } x \text {, y } \\ \text { 3) } 19.8 & <0.1 & \text { \# Integrated magnitude } \\ \text { 4) } 3.2 & 0.2 & \text { \# Re (effective radius) [pix] } \\ \text { 5) } 0.9 & 0.2 & \text { \# Sersic index } n \text { (de Vaucouleurs } n=4) \\ \text { 9) } 0.6 & 0.1 & \text { \# } & \text { Axis ratio (b/a) } \\ \text { 10) }-89 & 5 & \text { \# Position angle (PA) [deg: Up=0, Left=90] }\end{array}$

\# Component number: 9 - faint object (south)

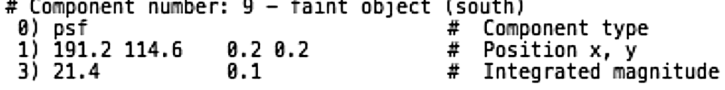

\# Component number: 10 - star (south west)

\# Component number: 10 - star (south west)

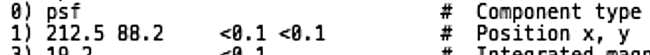

3) $19.2<0.1 \quad$ \# Integrated magnitude

\# Component number: 11 - star (east)

0) psf $118.7179 .1<0.1<0.1 \quad$ \# Component type

$\begin{array}{lll}\text { 1) } 118.7179 .1 & <0.1<0.1 & \text { \# } \\ \text { 3) } 19.2 & <0.1 & \# \text { Integrated magnitude }\end{array}$

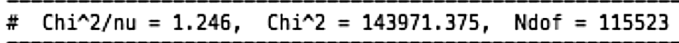

Figure A2. Best-fitting GALFIT parameters. Formal uncertainties (or upper limits) are indicated alongside the parameters, none of which was held fixed.

This paper has been typeset from a $\mathrm{T}_{\mathrm{E}} \mathrm{X} / \mathrm{L} \mathrm{T} \mathrm{T}_{\mathrm{E}} \mathrm{X}$ file prepared by the author. 